Draft Version SePtember 8, 2020

Typeset using $\mathrm{LAT}_{\mathrm{E}} \mathrm{X}$ twocolumn style in AASTeX63

\title{
A Theory for the Maximum Magnitude versus Rate of Decline (MMRD) Relation of Classical Novae
}

\author{
Izumi Hachisu, ${ }^{1}$ Hideyuki Saio, ${ }^{2}$ Mariko Kato,${ }^{3}$ Martin Henze, ${ }^{4}$ and Allen W. Shafter ${ }^{4}$ \\ ${ }^{1}$ Department of Earth Science and Astronomy, College of Arts and Sciences, The University of Tokyo, 3-8-1 Komaba, Meguro-ku, Tokyo \\ 153-8902, Japan \\ ${ }^{2}$ Astronomical Institute, Graduate School of Science, Tohoku University, Sendai 980-8578, Japan \\ ${ }^{3}$ Department of Astronomy, Keio University, Hiyoshi, Kouhoku-ku, Yokohama 223-8521, Japan \\ ${ }^{4}$ Department of Astronomy, San Diego State University, San Diego, CA 92182, USA
}

\begin{abstract}
We propose a theory for the MMRD relation of novae, using free-free emission model light curves built on the optically thick wind theory. We calculated $\left(t_{3}, M_{V, \max }\right)$ for various sets of $\left(\dot{M}_{\mathrm{acc}}, M_{\mathrm{WD}}\right)$, where $M_{V \text {,max }}$ is the peak absolute $V$ magnitude, $t_{3}$ is the 3 -mag decay time from the peak, and $\dot{M}_{\text {acc }}$ is the mass accretion rate on to the white dwarf (WD) of mass $M_{\mathrm{WD}}$. The model light curves are uniquely characterized by $x \equiv M_{\mathrm{env}} / M_{\mathrm{sc}}$, where $M_{\mathrm{env}}$ is the hydrogen-rich envelope mass and $M_{\mathrm{sc}}$ is the scaling mass at which the wind has a certain wind mass-loss rate. For a given ignition mass $M_{\mathrm{ig}}$, we can specify the first point $x_{0}=M_{\mathrm{ig}} / M_{\mathrm{sc}}$ on the model light curve, and calculate the corresponding peak brightness and $t_{3}$ time from this first point. Our $\left(t_{3}, M_{V \text {,max }}\right)$ points cover well the distribution of existing novae. The lower the mass accretion rate, the brighter the peak. The maximum brightness is limited to $M_{V, \max } \gtrsim-10.4$ by the lowest mass-accretion rate of $\dot{M}_{\text {acc }} \gtrsim 1 \times 10^{-11} M_{\odot} \mathrm{yr}^{-1}$. A significant part of the observational MMRD trend corresponds to the $\dot{M}_{\text {acc }} \sim 5 \times 10^{-9} M_{\odot} \mathrm{yr}^{-1}$ line with different WD masses. A scatter from the trend line indicates a variation in their mass-accretion rates. Thus, the global trend of an MMRD relation does exist, but its scatter is too large for it to be a precision distance indicator of individual novae.
\end{abstract}

Keywords: novae, cataclysmic variables — stars: individual (V1668 Cyg) — stars: winds

\section{INTRODUCTION}

A typical classical nova shows a rapid rise of optical brightness until its peak followed by a slow decline. There is a statistical trend that a faster decline nova shows a brighter optical peak. The scatter around the main trend, however, is not small. It has long been debated whether or not a meaningful relation actually exists (e.g., Mclaughlin 1945; Schmidt 1957; Cohen 1985; della Valle \& Livio 1995; Downes \& Duerbeck 2000; Kasliwal et al. 2011; Shafter et al. 2011; Cao et al. 2012; Shafter 2013; Shara et al. 2017; Schaefer 2018; Özdörmez et al. 2018; Selvelli \& Gilmozzi 2019; della Valle \& Izzo 2020). Such a relation is called the maximum magnitude versus rate of decline (MMRD) relation. If an MMRD relation exists and is a simple monotonic relation, it can be used to obtain the absolute peak brightness of a nova

izumi.hachisu@outlook.jp from the rate of decline, and thus, it would be a useful tool to obtain the distance to a nova. Although there are early attempts to theoretically explain the MMRD relation (e.g., Livio 1992), we need a convincing theoretical background to understand the main trend and large scatter of the existing MMRD distribution of novae.

A nova is a thermonuclear runaway event on a massaccreting white dwarf (WD). Hydrogen ignites and releases nuclear energy. The hydrogen-rich envelope expands to a giant size. The subsequent nova evolution was theoretically followed by Kato \& Hachisu (1994) based on the assumption of spherical symmetry. Strong optically-thick winds are accelerated deep inside the photosphere. The wind stops after a significant part of the hydrogen-rich envelope is ejected by the wind. The timescale of a nova in the early phase is determined by the wind mass-loss rate and the amount of the hydrogenrich envelope mass.

Kato \& Hachisu (1994) calculated optically thick winds in the decay phase of novae and obtained the 
photospheric radius $R_{\mathrm{ph}}$, temperature $T_{\mathrm{ph}}$, luminosity $L_{\mathrm{ph}}$, velocity $v_{\mathrm{ph}}$, and wind mass-loss rate $\dot{M}_{\text {wind }}$ against the decreasing envelope mass $M_{\mathrm{env}}$. Observationally, early spectra of novae are dominated by free-free emission (e.g., Ennis et al. 1977; Gallagher \& Ney 1976). Therefore, Hachisu \& Kato (2006) calculated free-free emission model light curves with $F_{\nu} \propto \dot{M}_{\text {wind }}^{2} /\left(v_{\mathrm{ph}}^{2} R_{\mathrm{ph}}\right)$, where $F_{\nu}$ is the flux at the frequency $\nu$. These model light curves well reproduce many nova light curves (e.g., Hachisu \& Kato 2006, 2010, 2016, 2018, 2019a,b).

The theoretical free-free emission light curves show a homologous shape independent of the WD mass and chemical composition. Hachisu \& Kato (2006) called this property of nova model light curves "the universal decline law." These properties, i.e., homologous and frequency independent shapes of light curves, indicate that the model light curves are expressed by a unique function of a parameter. We find that this parameter is the ratio of the envelope mass and the scaling envelope mass having a certain wind mass-loss rate, that is, $x \equiv$ $M_{\text {env }} / M_{\mathrm{sc}}$ as will be explained in Section 2.

Hachisu \& Kato (2010) found that two different model light curves, e.g., corresponding to the two different WD masses, can overlap each other if the timescale of one of them is squeezed by a factor of $f_{\mathrm{s}}$, i.e., $t / f_{\mathrm{s}}$. The normalization factor is $f_{\mathrm{s}}<1$ for a faster nova (corresponding to a more massive WD), and $f_{\mathrm{s}}>1$ for a slower nova (a less massive WD). Then the absolute $V$ brightnesses is normalized to be $M_{V}-2.5 \log f_{\mathrm{s}}$. Thus, the two different light curves overlap each other in the $\left(t / f_{\mathrm{s}}\right)-\left(M_{V}-2.5 \log f_{\mathrm{s}}\right)$ plane (see, e.g., Figures 48 and 49 of Hachisu \& Kato 2018). Hachisu \& Kato (2019b) reformulated this property: if the $V$ light curve of a template nova (time $t$ ) overlaps with that of a target nova (time $t^{\prime}=t / f_{\mathrm{s}}$ ), we have the relation

$$
\begin{aligned}
\left(M_{V}[t]\right)_{\text {template }} & =\left(M_{V}^{\prime}\left[t^{\prime}\right]\right)_{\text {target }} \\
& =\left(M_{V}\left[t / f_{\mathrm{s}}\right]-2.5 \log f_{\mathrm{s}}\right)_{\text {target }},
\end{aligned}
$$

where $M_{V}[t]$ is the original absolute $V$ brightness and $M_{V}^{\prime}\left[t^{\prime}\right]$ is the time-normalized brightness after timenormalization of $t^{\prime}=t / f_{\mathrm{s}}$. This property was calibrated on many novae (e.g., Hachisu \& Kato 2016, 2018, 2019a).

Hachisu \& Kato (2010) also presented a theoretical explanation of the MMRD relation based on the universal decline law and Equation (1). Their interpretation on the main trend of the MMRD distribution is that V1668 Cyg is a typical classical nova, and that the novae having the same time-normalized light curves (i.e., the same normalized peak brightnesses) as that of V1668 Cyg but the different WD masses form the main trend line in the $\left(\log t_{3}\right)-M_{V, \max }$ diagram, i.e., $M_{V, \max }=$
$2.5 \log t_{3}-11.94$ (Equation (25) in Hachisu \& Kato 2016). Here, $t_{3}$ is the 3 -mag decay time from the $V$ peak and $M_{V \text {,max }}$ is the absolute $V$ peak magnitude. This main trend line is located in the middle of the observational MMRD distribution of novae in the $\left(\log t_{3}\right)-$ $M_{V, \text { max }}$ diagram and its peak becomes brighter along Equation (1) with the decreasing $f_{\mathrm{s}}$. On this main trend line, a faster decline nova with a shorter $t_{3}$ time (smaller $f_{\mathrm{s}}$ ) corresponds to a more massive WD while a slower decline nova with a longer $t_{3}$ time (larger $f_{\mathrm{s}}$ ) does to a less massive WD.

In the present work, we clarify the physics of MMRD points. Then, we explain the main trend of MMRD relation as a typical $\dot{M}_{\text {acc }}$ with the different $M_{\mathrm{WD}}$ 's. The scatter from the main trend line is explained by the difference of $\dot{M}_{\text {acc }}$ from a typical $\dot{M}_{\text {acc }}$.

Our paper is organized as follows. First we propose several timescaling laws and clarify the physics of MMRD relation in Section 2. Then, we approximate these timescaling laws with analytic expressions in Section 3 , which simplify the calculations of $\left(t_{3}, M_{V, \text { max }}\right)$. In Section 4, we explain our theoretical $\left(t_{3}, M_{V \text {,max }}\right)$ relation on the base of $\left(\dot{M}_{\mathrm{acc}}, M_{\mathrm{WD}}\right)$, the main characteristic properties of cataclysmic binaries. Discussion and our conclusions are given in Sections 5 and 6, respectively. We tabulate our numerical results in Appendix A.

\section{TIMESCALING LAW OF FREE-FREE EMISSION MODEL LIGHT CURVES}

Kato \& Hachisu (1994) calculated envelope solutions of wind mass-loss for various WD masses (ranging from $0.5 M_{\odot}$ to $1.38 M_{\odot}$ ) and chemical compositions. They provide the wind mass-loss rate $\dot{M}_{\text {wind }}$, photospheric temperature $T_{\mathrm{ph}}$, velocity $v_{\mathrm{ph}}$, and radius $R_{\mathrm{ph}}$ for a specific envelope mass $M_{\mathrm{env}}$ and WD mass $M_{\mathrm{WD}}$. Hachisu \& Kato $(2006,2010)$ calculated the nova optical and infrared (IR) light curves based mainly on the free-free emission model of winds. We plot such examples in Figures 1, 2, 3, and 4 for the chemical composition of typical $\mathrm{CO}$ novae, i.e., $\mathrm{CO}$ nova 3 (CO3; Hachisu \& Kato 2016), each element of which is $\left(X, Y, Z, X_{\mathrm{C}}, X_{\mathrm{O}}\right)=(0.45,0.18,0.02,0.15,0.20)$ by mass weight.

\subsection{Brightnesses of Optical/IR Light Curves}

Hachisu \& Kato (2006, 2010) calculated the nova optical/IR light curves based mainly on the free-free emission model of winds. Their free-free emission flux is approximately calculated from

$$
F_{\nu}\left(t: M_{\mathrm{WD}}\right)=A_{\mathrm{ff}} \frac{\dot{M}_{\mathrm{wind}}^{2}}{v_{\mathrm{ph}}^{2} R_{\mathrm{ph}}},
$$




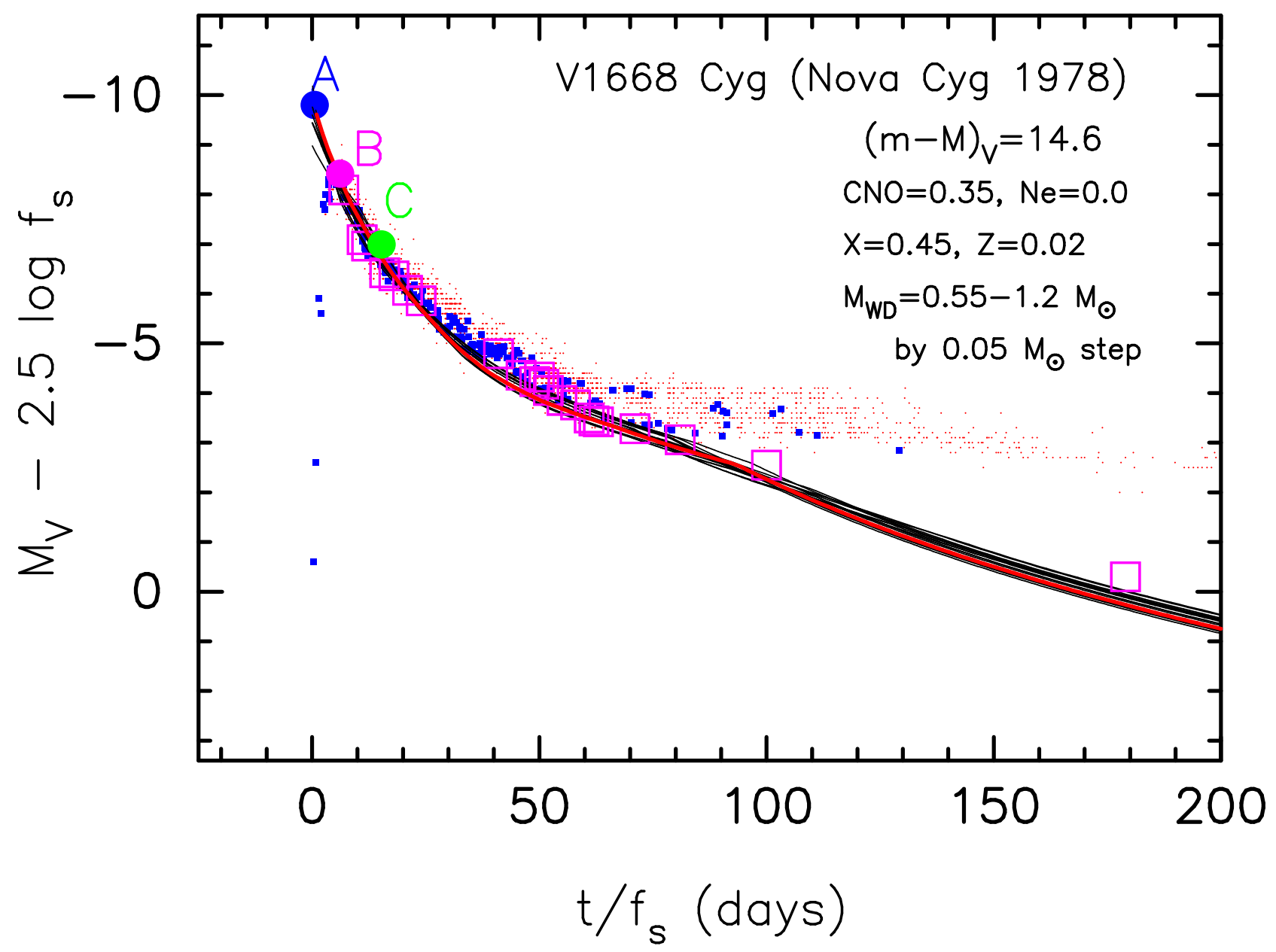

Figure 1. Comparison of our model $V$ light curves with the V1668 Cyg light curve. A $0.98 M_{\odot}$ WD (CO3) model (solid red line) reasonably reproduces the V1668 Cyg optical $y$ (unfilled magenta squares) and $V$ (filled blue squares) light curves. We add the visual magnitudes (red dots) of V1668 Cyg. The distance modulus in $V$ band of $\mu_{V} \equiv(m-M)_{V}=14.6$ is taken from Hachisu \& Kato (2019a). The data are all the same as Figure 46 of Hachisu \& Kato (2018). Free-free emission model $V$ light curves for $0.55,0.60,0.65,0.70,0.75,0.80,0.85,0.90,0.95,1.0,1.05,1.1,1.15$, and $1.20 M_{\odot}$ WDs (solid black lines) are plotted in the $\left(t / f_{\mathrm{s}}\right)-\left(M_{V}-2.5 \log f_{\mathrm{s}}\right)$ plane. The time-normalization factor $f_{\mathrm{s}}$ of each model, tabulated in Table 3 of Hachisu \& Kato (2016), is measured against that of the V1668 Cyg light curves. We place three points, A, B, and C, on the model $V$ light curve $\left(0.98 M_{\odot}\right)$, corresponding to three different initial envelope masses, $M_{\mathrm{env}, 0}=1.8 \times 10^{-5} M_{\odot}, 1.4 \times 10^{-5} M_{\odot}$, and $0.94 \times 10^{-5} M_{\odot}$, respectively. Point B is the optical peak of V1668 Cyg, $m_{V, \max }=6.2\left(M_{V, \max }=6.2-14.6=-8.4\right)$.

where they assumed $A_{\mathrm{ff}}$ to be a constant among various novae. The effects of the electron temperature, ionization degree, and chemical composition on the freefree flux are included through the envelope solutions, i.e., the wind mass-loss rate $\dot{M}_{\text {wind }}$, photospheric velocity $v_{\mathrm{ph}}$, and photospheric radius $R_{\mathrm{ph}}$. Hachisu \& Kato (2010, 2016) determined $A_{\text {ff }}$ by fitting the $0.98 M_{\odot}$ WD (CO3) model light curve with the V1668 Cyg light curves, where the distance modulus in $V$ band $\mu_{V} \equiv(m-M)_{V}=14.6$ as shown in Figure 1.

Figure 1 shows our free-free emission model light curves for $0.55,0.60,0.65,0.70,0.75,0.80,0.85,0.90$, $0.95,1.0,1.05,1.1,1.15$, and $1.2 M_{\odot}$ WDs (CO3). All the model light curves overlap well with each other in the $\left(t / f_{\mathrm{s}}\right)-\left(M_{V}-2.5 \log f_{\mathrm{s}}\right)$ plane. Hachisu \& Kato (2006) called this property the universal decline law. Here, the timescaling factor $f_{\mathrm{s}}$ is measured against the timescale of V1668 Cyg. The light curve of this nova is well reproduced with a $0.98 M_{\odot} \mathrm{WD}$ (CO3) model (solid red line). See Hachisu \& Kato (2016, 2019a,b) for the model light curve fitting of V1668 Cyg.

To deeply understand the physics of nova light curves, we break the scaling process shown in Figure 1 into two steps. We plot the first step in Figure 2(a)(b) for three WD mass models of $0.9 M_{\odot}$ (green), $1.0 M_{\odot}$ (red), and $1.1 M_{\odot}$ (blue), that is, the free-free flux parameter $\dot{M}_{\text {wind }}^{2} /\left(v_{\mathrm{ph}}^{2} R_{\mathrm{ph}}\right)$ against $\dot{M}_{\text {wind }}$. Then, we plot the second step in Figure 2(c)(d), that is, the wind massloss rate $\dot{M}_{\text {wind }}$ against the real time $t$ and normalized time $t / f_{\mathrm{s}}$, respectively. The third step is the combina- 

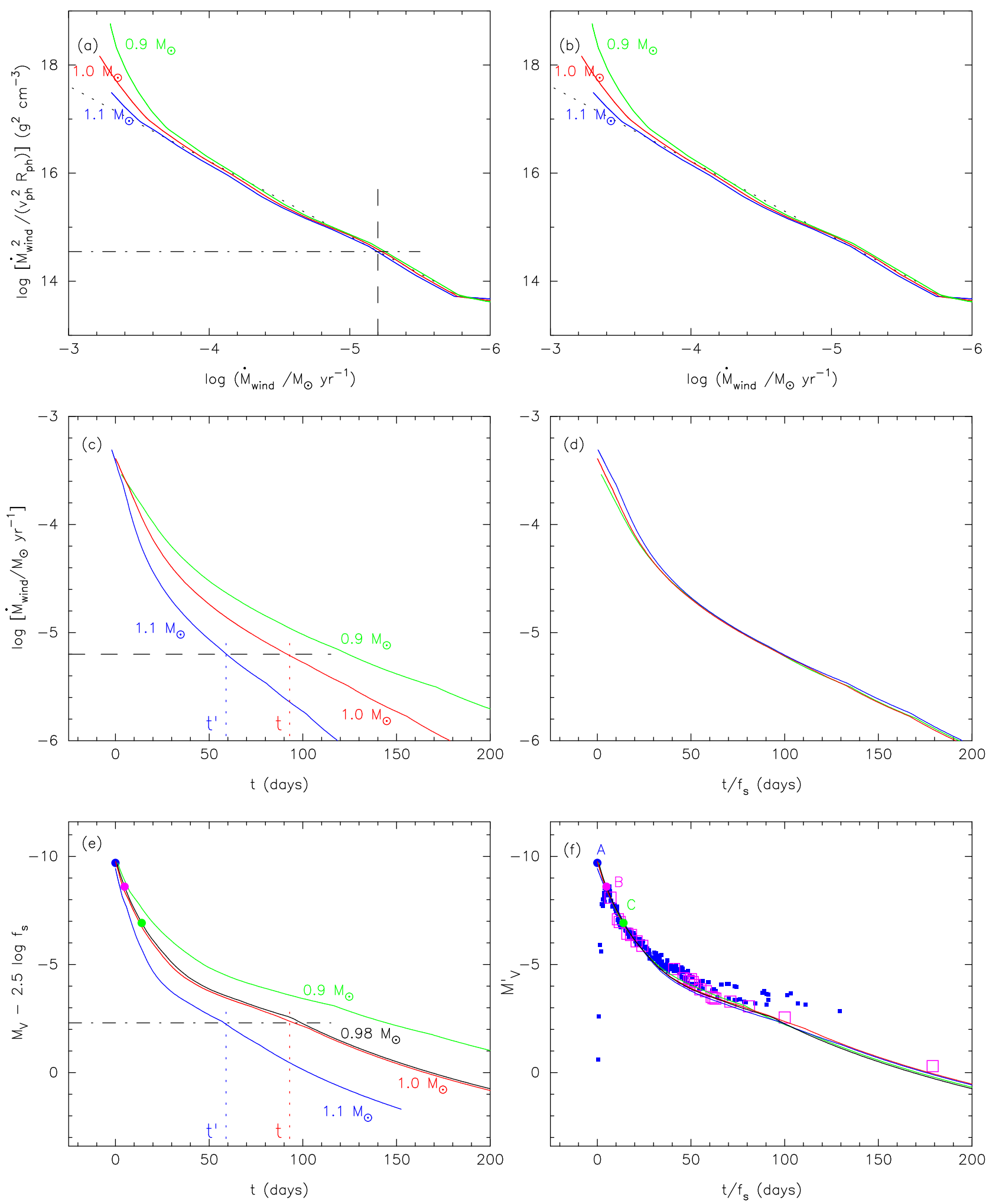

Figure 2. (a)(b) The free-free flux parameter of $\dot{M}_{\text {wind }}^{2} /\left(v_{\mathrm{ph}}^{2} R_{\mathrm{ph}}\right)$ versus wind mass-loss rate $\dot{M}_{\text {wind }}$ for $1.1 M_{\odot}$ (blue), $1.0 M_{\odot}$ (red), and $0.9 M_{\odot}$ (green) WD (CO3) models, which are the same models as in Figure 1. The three (blue, red, and green) lines almost overlap each other. The dotted lines indicate the global trend of these three lines. (c)(d) The wind mass-loss rates are plotted against the real time $t$ and the normalized time $t / f_{\mathrm{s}}$, respectively, for the same three WD mass models. (e)(f) Free-free emission model $V$ light curves for the $0.90 M_{\odot}$ (green), $0.98 M_{\odot}$ (black), $1.0 M_{\odot}$ (red), and $1.1 M_{\odot}$ (blue) WDs are plotted against the real time $t$ and the normalized time $t / f_{\mathrm{s}}$, respectively. In panel (e), the right edge of $1.1 M_{\odot}$ light curve (blue line) corresponds to the end of wind phase. Note that the ordinate in panel (f) is $M_{V}^{\prime}\left[t^{\prime}\right]$ in Equation (1), corresponding to the abscissa of $t^{\prime}=t / f_{\mathrm{s}}$ while the ordinate in panel (e) is $M_{V}[t]-2.5 \log f_{\mathrm{s}}$ because the abscissa is $t$. The symbols in panels (e) and (f) are the same as those in Figure 1. See the text for more details. 
tion of the first and second steps, which is plotted in Figure 2(e)(f). Here, we add a $0.98 M_{\odot}$ WD (CO3) model (black line). Note that the ordinate in panel (e) is $M_{V}[t]-2.5 \log f_{\mathrm{s}}$ because the abscissa is $t$ while the ordinate in panel (f) is $M_{V}^{\prime}\left[t / f_{\mathrm{s}}\right]\left(=M_{V}\left[t / f_{\mathrm{s}}\right]-2.5 \log f_{\mathrm{s}}\right)$.

The right column, Figure 2(b)(d)(f), are the same as those in the left column, but in the normalized timescale, $t / f_{\mathrm{s}}$. Note that Figure 2(a) and 2(b) are essentially the same because the time does not explicitly appear. Combining Figure 2(b) with 2(d), we obtain Figure 2(f). The proportionality constant $A_{\mathrm{ff}}$ is determined by fitting the $0.98 M_{\odot}$ WD model light curve with the V1668 Cyg light curve. Figure 2(f) is essentially the same as Figure 1.

The overlapping of envelope solutions in Figure 2(a)(b) directly means that, when the wind mass-loss rates are the same, the fluxes of free-free emission are the same irrespective of the WD mass and chemical composition. We express this with a function of $F_{\nu}\left(\dot{M}_{\text {wind }}\right)$. This expression does not explicitly include the WD mass but $F_{\nu}\left(t: M_{\mathrm{WD}}\right)$ of Equation (2) depends on the WD mass through the wind mass-loss rate $\dot{M}_{\text {wind }}$ as shown in Figure 2(c).

After the optical maximum, the free-free flux decreases as the wind mass-loss rate drops. This relation, $F_{\nu}\left(\dot{M}_{\text {wind }}\right)$, is common among the various WD masses and chemical compositions. In the real timescale of Figure 2(c) and 2(e), however, the wind mass-loss rate and flux decrease more rapidly in more massive WDs. To clarify the difference in the timescale between the $1.0 M_{\odot}$ and $1.1 M_{\odot}$ WDs, we designate their times $t$ and $t^{\prime}$, respectively. For example, we plot a relation between $t$ and $t^{\prime}$ at the vertical dashed line of $\log \left(\dot{M}_{\text {wind }} / M_{\odot} \mathrm{yr}^{-1}\right)=-5.2$ in Figure $2(\mathrm{a})$, which corresponds to the horizontal dashed line in Figure 2(c). The two dotted lines show the positions of $t$ and $t^{\prime}$. This $t$ and $t^{\prime}$ relation similarly holds for the horizontal dash-dotted lines of the free-free flux parameter $\dot{M}_{\text {wind }}^{2} /\left(v_{\mathrm{ph}}^{2} R_{\mathrm{ph}}\right)$ and $M_{V}-2.5 \log f_{\mathrm{s}}$ in Figure $2(\mathrm{a})$ and $2(\mathrm{e})$.

We formulate the conversion from $(t, F(t))$ to $\left(t^{\prime}, F^{\prime}\left(t^{\prime}\right)\right)$ by the time-normalization of $t^{\prime}=t / f_{\mathrm{s}}$, that is,

$$
F^{\prime}\left(t^{\prime}\right)=f_{\mathrm{s}} F\left(t / f_{\mathrm{s}}\right) .
$$

This simply means that, if the timescale is squeezed by ten times $\left(t^{\prime}=t / 10\right)$, the squeezed flux becomes larger $\left(F^{\prime}=10 F\right)$ because of energy conservation, i.e., $\Delta E=$ $F^{\prime}\left(t^{\prime}\right) \Delta t^{\prime}=f_{\mathrm{s}} F\left(t^{\prime}\right) \Delta t^{\prime}=F\left(t / f_{\mathrm{s}}\right) \Delta t$. In other words, we observe the same outburst with two different temporal scalings, $t$ and $t^{\prime}$. Then, the flux is different between these two systems, $F(t)$ and $F^{\prime}\left(t^{\prime}\right)$, while the energy is the same, $\Delta E$, during the same intrinsic time-interval, $\Delta t^{\prime}=\Delta t / f_{\mathrm{s}}$, at the same intrinsic time, $t^{\prime}=t / f_{\mathrm{s}}$.
From Figure 2(a)(c), for example, we obtain

$$
\begin{aligned}
F\left(t: 1.0 M_{\odot}\right) & =A_{\mathrm{ff}} \frac{\dot{M}_{\mathrm{wind}}^{2}}{v_{\mathrm{ph}}^{2} R_{\mathrm{ph}}}=F^{\prime}\left(t^{\prime}: 1.1 M_{\odot}\right) \\
& =f_{\mathrm{s}} F\left(t / f_{\mathrm{s}}: 1.1 M_{\odot}\right) .
\end{aligned}
$$

Then, we convert the flux of Equation (4) to the absolute $V$ magnitude as

$$
\begin{aligned}
& M_{V}\left(t: 1.0 M_{\odot}\right)=M_{V}^{\prime}\left(t^{\prime}: 1.1 M_{\odot}\right) \\
= & M_{V}\left(t / f_{\mathrm{s}}: 1.1 M_{\odot}\right)-2.5 \log f_{\mathrm{s}},
\end{aligned}
$$

where $M_{V}$ is the absolute $V$ magnitude of the free-free emission light curve. The same equation holds for the $0.9 M_{\odot}$ and $1.0 M_{\odot}$ WDs. Thus, we derive Equation (1).

\subsection{Timescales of Optical/IR Light Curves}

The hydrogen-rich envelope mass decreases with time. We plot $-\dot{M}_{\text {env }}$ against $M_{\text {env }}$ for the three WD masses of $1.1 M_{\odot}$ (blue), $1.0 M_{\odot}$ (red), and $0.9 M_{\odot}$ (green) in Figure 3(a). Here, the decreasing rate of the envelope mass is the summation of the wind mass-loss rate and mass-decreasing rate by nuclear burning, i.e.,

$$
-\dot{M}_{\text {env }}=\dot{M}_{\text {wind }}+\dot{M}_{\text {nuc }} \text {. }
$$

Time goes on from the upper-right to lower-left of each line. The decrease in the brightness corresponds to the same color line in Figure 2(e)(f). The starting point is somewhere on the line at $M_{\mathrm{env}}=M_{\mathrm{env}, 0}=M_{\mathrm{ig}}$, which corresponds to the maximum brightness of a nova outburst. Here, we specify the envelope mass at the maximum brightness by $M_{\mathrm{env}, 0}$ which we regard to be equal to the ignition mass, $M_{\mathrm{ig}}$. The ignition mass is defined by the hydrogen-rich envelope mass at the start of hydrogen burning. The wind mass-loss rate $\dot{M}_{\text {wind }}$ decreases with decreasing envelope mass $M_{\mathrm{env}}$, and finally vanishes when the envelope mass reaches the critical envelope mass $M_{\mathrm{cr}}$ required to drive a wind. After that, the envelope mass decreases slowly by nuclear burning. The nova ends when the nuclear burning extinguishes at the bottom of each line. The sudden flattening on each line corresponds to the end of the wind phase.

If we normalize the envelope mass by each scaling mass, $M_{\mathrm{env}} / M_{\mathrm{sc}}$, these three lines almost perfectly overlap each other for $\log \left(-\dot{M}_{\mathrm{env}} / M_{\odot} \mathrm{yr}^{-1}\right) \geq-4.7$ as depicted by the horizontal dashed line as shown in Figure 3(b). Here, we define the scaling mass by each envelope mass having $\log \left(-\dot{M}_{\text {env }} / M_{\odot} \mathrm{yr}^{-1}\right)=-4.7$ denoted by the vertical dashed line in Figure 3(b). We add other two WD masses, $0.8 M_{\odot}$ (magenta) and $1.2 M_{\odot}$ (black). We show only five WD masses in this figure, but obtained 

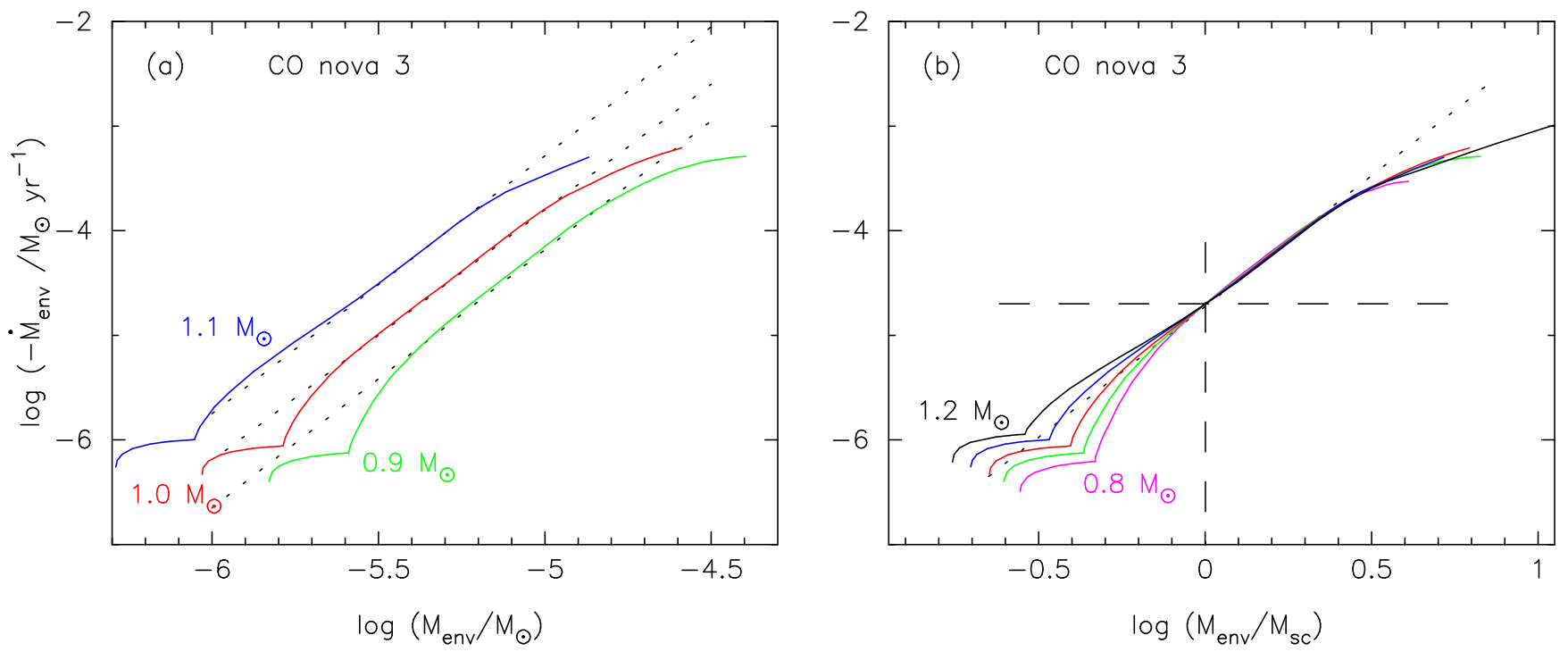

Figure 3. (a) The envelope mass decreasing rate versus hydrogen-rich envelope mass of optically thick wind (or static) solutions for three WD masses with the chemical composition of CO nova 3 (CO3). The solid blue, red, and green lines denote $1.1 M_{\odot}$, $1.0 M_{\odot}$, and $0.9 M_{\odot}$ WD models, respectively. The break of each line corresponds to the $M_{\text {cr }}$ at which optically thick winds stop. The dotted lines indicate the global trends of $\dot{M}_{\text {env }}-M_{\text {env }}$ relations. (b) The ordinate is the same, but the horizontal axis is scaled by each scaling mass $M_{\mathrm{sc}}$, where the scaling mass is defined by each envelope mass having $\log \left(-\dot{M}_{\mathrm{env}} / M_{\odot} \mathrm{yr}^{-1}\right)=-4.7$. Two other WD mass models are added, i.e., $0.8 M_{\odot}$ (magenta) and $1.2 M_{\odot}$ (black). The horizontal dashed line denotes the envelope mass decreasing rate of $\log \left(-\dot{M}_{\mathrm{env}} / M_{\odot} \mathrm{yr}^{-1}\right)=-4.7$ while the vertical dashed line indicates each scaling mass.

the similar tendency of envelope solutions for other WD masses (ranging from $0.6 M_{\odot}$ to $1.3 M_{\odot}$ ) with the same or different chemical compositions (see, e.g., Figure 6 of Kato \& Hachisu 1994).

Overlapping of the five lines means that we can express $-\dot{M}_{\text {env }}(x) \approx \dot{M}_{\text {wind }}(x)$ as a function of single parameter of $x$, i.e.,

$$
x \equiv \frac{M_{\mathrm{env}}}{M_{\mathrm{sc}}}
$$

independently of the WD mass or chemical composition, while the $M_{\mathrm{sc}}$ itself depends both on the $M_{\mathrm{WD}}$ and chemical composition, but is almost independent of the mass accretion rate for $\dot{M}_{\text {acc }} \lesssim 1 \times 10^{-8} M_{\odot} \mathrm{yr}^{-1}$. For a larger mass accretion rate of $\dot{M}_{\text {acc }} \gtrsim 3 \times 10^{-8} M_{\odot} \mathrm{yr}^{-1}$, the WD radius is slightly larger compared with that of the cold core. As a result, the $M_{\text {sc }}$ for $\dot{M}_{\text {acc }} \gtrsim$ $3 \times 10^{-8} M_{\odot} \mathrm{yr}^{-1}$ is slightly larger than that for the cold core of $\dot{M}_{\text {acc }} \lesssim 1 \times 10^{-8} M_{\odot} \mathrm{yr}^{-1}$. In the present paper, however, we assume that $M_{\mathrm{sc}}$ is independent of the mass accretion rate.

The increase with $M_{\text {env }} / M_{\mathrm{sc}}$ in the wind mass-loss rate seems to saturate at the upper-right end of the three lines. In this region, $-\dot{M}_{\text {env }} \approx \dot{M}_{\text {wind }}$ because $\dot{M}_{\text {nuc }} \ll \dot{M}_{\text {wind }}$. The numerical method adopted by Kato \& Hachisu (1994) requires convergence of numerical iterations to precisely obtain the wind mass-loss rate. The convergence becomes very slow or fails near the region where the lines seem to saturate. Therefore, we suppose that the true wind mass-loss rate does not saturate but increases along the dotted line.

Then, the elapsed time is calculated from the decreasing rate of the envelope mass, that is,

$$
\begin{aligned}
t & =\int \frac{d M_{\mathrm{env}}}{\dot{M}_{\mathrm{env}}}=M_{\mathrm{sc}} \int \frac{d\left(M_{\mathrm{env}} / M_{\mathrm{sc}}\right)}{\dot{M}_{\mathrm{env}}} \\
& =\frac{M_{\mathrm{sc}}\left(M_{\mathrm{WD}}\right)}{M_{\mathrm{sc}, 0}} \tau=f_{\mathrm{s}} \tau,
\end{aligned}
$$

where $M_{\mathrm{sc}, 0}$ is a given envelope mass (we adopt $M_{\mathrm{sc}, 0}=$ $0.448 \times 10^{-5} M_{\odot}$ later in Equation (11)) and

$$
\tau \equiv M_{\mathrm{sc}, 0} \int \frac{d\left(M_{\mathrm{env}} / M_{\mathrm{sc}}\right)}{\dot{M}_{\mathrm{env}}}=M_{\mathrm{sc}, 0} \int \frac{d x}{\dot{M}_{\mathrm{env}}(x)} .
$$

Therefore, we obtain

$$
f_{\mathrm{s}}=\frac{M_{\mathrm{sc}}}{M_{\mathrm{sc}, 0}}
$$

from the last equality in Equation (8). We explicitly write $M_{\mathrm{sc}}\left(M_{\mathrm{WD}}\right)$ because the scaling mass depends on the WD mass, i.e., a function of $M_{\mathrm{WD}}$ for a given chemical composition, as shown later in Figure 7(a). The overlapping of lines for $\log \left(-\dot{M}_{\text {env }} / M_{\odot} \mathrm{yr}^{-1}\right) \geq-4.7$ in Figure 3(b) guarantees that each timescale is proportional to $f_{\mathrm{s}} \propto M_{\mathrm{sc}}$. The fact that the envelope mass decreasing rate $\dot{M}_{\text {env }}(x)$ is approximately a unique function of $x \equiv M_{\mathrm{env}} / M_{\mathrm{sc}}$ and that the timescale is propor- 
tional to $f_{\mathrm{s}} \propto M_{\mathrm{sc}}$ are the first important conclusions of the present paper.

\subsection{Universal Decline Law and Peak Brightness}

Figure 4 shows the time-normalized absolute $V$ magnitude, $M_{V}-2.5 \log f_{\mathrm{s}}$, against the scaled envelope mass, $M_{\text {env }} / M_{\text {sc }}$, for $0.9 M_{\odot}$ (green), $0.98 M_{\odot}$ (black), $1.0 M_{\odot}$ (red), and 1.1 $M_{\odot}$ (blue) WDs. These four lines almost overlap with each other. Therefore, $f_{\mathrm{s}} F_{\nu}(x)$ is approximately a unique function of $x=M_{\mathrm{env}} / M_{\mathrm{sc}}$ irrespective of the WD mass and chemical composition. This is the second important conclusion of the present paper.

This can be understood as follows: we obtain the universal decline law in Figure 2(f) combining Figure 2(b) and Figure 2(d). Similarly, we obtain the overlap of each line in Figure 4 by combining Figure 2(b) and Figure $3(\mathrm{~b})$. This is because $-\dot{M}_{\text {env }} \approx \dot{M}_{\text {wind }}$ for $\log \left(M_{\mathrm{env}} / M_{\mathrm{sc}}\right)=\log x \geq-0.1$ in Figure $3(\mathrm{~b})$.

The blue/green lines slightly deviate from the other lines for $\log \left(M_{\text {env }} / M_{\mathrm{sc}}\right) \gtrsim 0.5$. We suppose that these deviations are due to the effect of numerical convergence of iterations as mentioned above in Section 2.2. The blue line $\left(1.1 M_{\odot} \mathrm{WD}\right)$ happens to be located below the other lines of $0.98 M_{\odot}$ (black) and $1.0 M_{\odot}($ red) WDs while the green line $\left(0.9 M_{\odot}\right)$ slightly diverges upward at $\log \left(M_{\mathrm{env}} / M_{\mathrm{sc}}\right) \gtrsim 0.5$. We assume that the true $M_{V}-$ $2.5 \log f_{\mathrm{s}}$ for the $1.1 M_{\odot}$ and $0.9 M_{\odot}$ WDs follow the dotted line like the other two lines.

Figure 1 shows our free-free emission model light curves for $0.55,0.60,0.65,0.70,0.75,0.80,0.85,0.90$, $0.95,1.0,1.05,1.1,1.15$, and $1.2 M_{\odot}$ WDs (CO3). The timescale of each WD is measured against that of the $0.98 M_{\odot} \mathrm{WD}(\mathrm{CO} 3)$, i.e.,

$$
\begin{aligned}
f_{\mathrm{s}}\left(M_{\mathrm{WD}}\right) & =\frac{M_{\mathrm{sc}}\left(M_{\mathrm{WD}}\right)}{M_{\mathrm{sc}}\left(0.98 M_{\odot}, \mathrm{CO} 3\right)} \\
& =\frac{M_{\mathrm{sc}}\left(M_{\mathrm{WD}}\right)}{0.448 \times 10^{-5} M_{\odot}} .
\end{aligned}
$$

We confirmed that the timescaling factor $f_{\mathrm{s}}$ defined by Equation (10) or (11) is in good agreement with the timescaling factor $f_{\mathrm{s}}$ defined directly by the light curves of the universal decline law in Figure 1.

In short, the brightness of the universal decline law in Figure 1 can be specified by the two parameters, $f_{\mathrm{s}}$ and $\tau \equiv t / f_{\mathrm{s}}$, where $f_{\mathrm{s}}$ is related to $M_{\mathrm{sc}}$ through Equation (11) and $\tau$ is related to $x$ with $M_{V}[\tau]-2.5 \log f_{\mathrm{s}}=$ $M_{V}[x]-2.5 \log f_{\mathrm{s}}$ in Figure 2(f) and Figure 4. Thus, the two parameter set of $\left(f_{\mathrm{s}}, \tau\right)$ is equivalent to the set of $\left(M_{\mathrm{sc}}, x\right)$.

In Figure 1, the optical peak of V1668 Cyg corresponds to point B. Point A (C) indicates a much brighter (fainter) nova. These points A, B, and $\mathrm{C}$ are also plotted
Table 1. Scaling Masses for CO Novae ${ }^{a}$

\begin{tabular}{llll}
\hline \hline$M_{\mathrm{WD}}$ & $M_{\mathrm{sc}}(\mathrm{CO} 2)$ & $M_{\mathrm{sc}}(\mathrm{CO} 3)$ & $M_{\mathrm{sc}}(\mathrm{CO} 4)$ \\
$\left(M_{\odot}\right)$ & $\left(10^{-5} M_{\odot}\right)$ & $\left(10^{-5} M_{\odot}\right)$ & $\left(10^{-5} M_{\odot}\right)$ \\
\hline 0.55 & 2.80 & 2.74 & 3.34 \\
0.60 & 2.22 & 2.17 & 2.63 \\
0.65 & 1.84 & 1.80 & 2.16 \\
0.70 & 1.53 & 1.50 & 1.80 \\
0.75 & 1.21 & 1.18 & 1.41 \\
0.80 & 0.961 & 0.935 & 0.112 \\
0.85 & 0.769 & 0.746 & 0.887 \\
0.90 & 0.617 & 0.598 & 0.709 \\
0.95 & 0.514 & 0.498 & 0.588 \\
0.98 & 0.462 & 0.448 & $\ldots$ \\
1.00 & 0.430 & 0.416 & 0.491 \\
1.05 & 0.339 & 0.329 & 0.385 \\
1.10 & 0.270 & 0.260 & 0.304 \\
1.15 & 0.215 & 0.207 & 0.241 \\
1.20 & 0.171 & 0.165 & 0.192 \\
\hline \multicolumn{5}{c}{ chemical composition of the hydrogen-rich } \\
envelope is assumed to be those of "CO \\
nova 2", "CO nova 3", and "CO nova 4" \\
in Table 2 of Hachisu \& Kato (2006), i.e., \\
(X, $\left.Y, Z, X_{\mathrm{C}}, X_{\mathrm{O}}\right)$ & $=(0.35,0.33,0.02,0.10,0.20)$, \\
$(0.45,0.18,0.02,0.15,0.20)$, & and \\
$(0.55,0.23,0.02,0.10,0.10)$, respectively.
\end{tabular}

in Figure 2(e)(f) against the real time $t$ and normalized time $\tau=t / f_{\mathrm{s}}$, respectively. Points $\mathrm{A}, \mathrm{B}$, and $\mathrm{C}$ correspond to different initial envelope masses as shown in Figure 4. Point A, the brightest one, has $M_{V, \max }=-9.7$ and $M_{\mathrm{env}, 0}=1.8 \times 10^{-5} M_{\odot}\left(x_{0} \equiv M_{\mathrm{env}, 0} / M_{\mathrm{sc}}=4.0\right)$. Point B has $M_{V, \max }=-8.4$ and $M_{\text {env }, 0}=1.4 \times 10^{-5} M_{\odot}$ $\left(x_{0}=3.1\right)$. Point $\mathrm{C}$ has $M_{V, \max }=-6.9$ and $M_{\mathrm{env}, 0}=$ $0.94 \times 10^{-5} M_{\odot}\left(x_{0}=2.1\right)$. For a given $\mathrm{WD}$ mass $\left(0.98 M_{\odot}\right)$, the brighter peak corresponds to a larger ignition mass. Here, we approximate the initial envelope mass $M_{\mathrm{env}, 0}$ by the ignition mass $M_{\mathrm{ig}}$ of a nova outburst. In general, the ignition mass depends on the mass accretion rate on to the WD (e.g., Nomoto 1982; Townsley \& Bildsten 2004; Wolf et al. 2013a,b). The lower the mass accretion rate, the larger the ignition mass for a given WD mass. Thus, the nova is brighter for a smaller mass-accretion rate even if the WD mass is the same.

\section{APPROXIMATE ANALYTIC RELATIONS BETWEEN VARIOUS PHYSICAL QUANTITIES}

The peak brightness of a nova is calculated from the initial envelope mass, $M_{\text {env, } 0}$, as shown in Figure 4 . We make an approximate analytic relation for this (black dotted line), i.e.,

$$
M_{V}-2.5 \log f_{\mathrm{s}}=-9.5 \log \left(\frac{M_{\mathrm{env}}}{M_{\mathrm{sc}}}\right)-3.73 .
$$




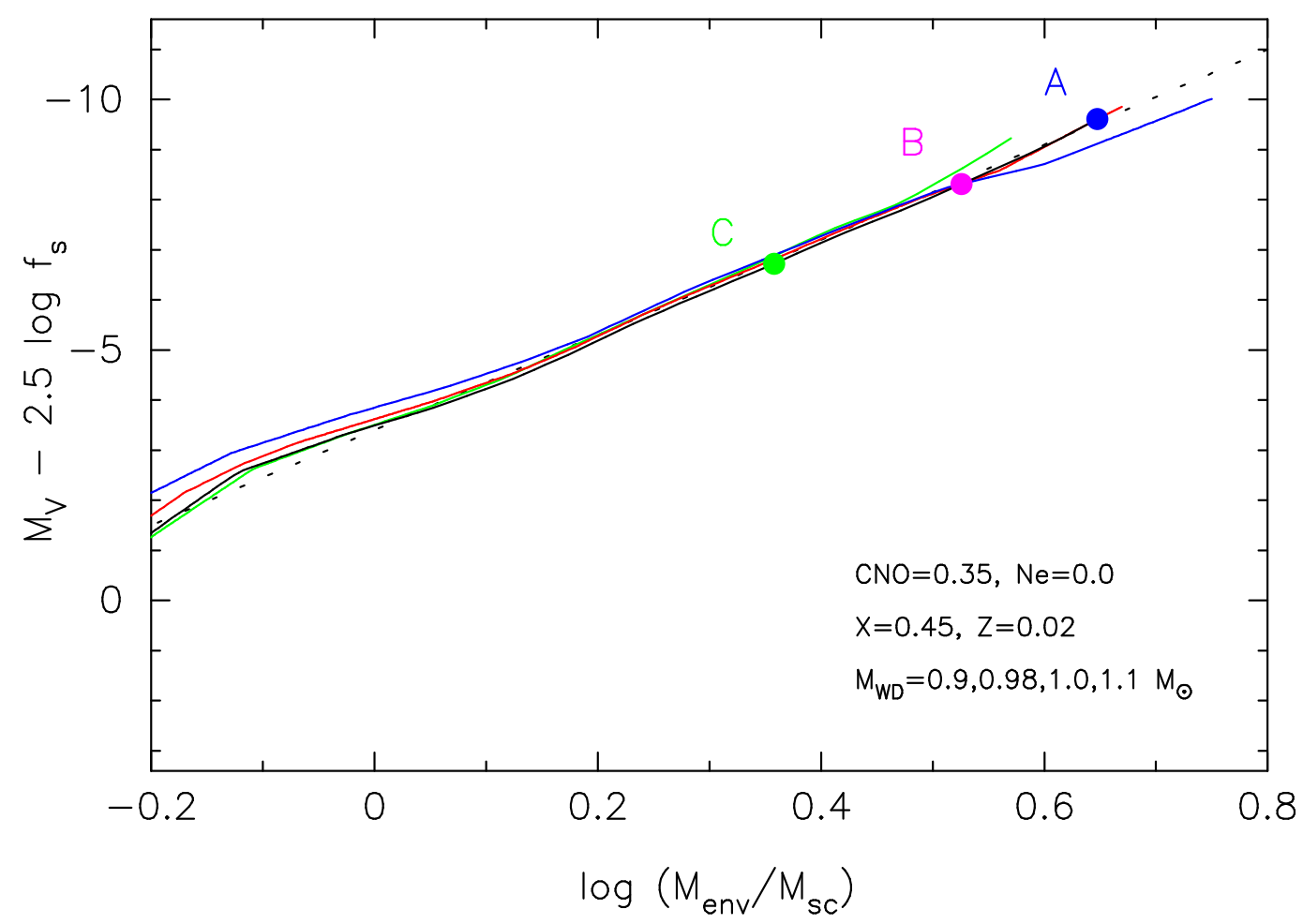

Figure 4. Free-free emission model $V$ light curves are plotted against $M_{\text {env }} / M_{\text {sc }}$ for the $0.90 M_{\odot}$ (green), $0.98 M_{\odot}$ (black), $1.0 M_{\odot}\left(\right.$ red), and $1.1 M_{\odot}$ (blue) WDs. The absolute $V$ magnitude is calibrated with the V1668 Cyg light curve (Hachisu \& Kato 2016). The three points of $\mathrm{A}, \mathrm{B}$, and $\mathrm{C}$ are the same as those in Figure 1 and specified by $x_{0} \equiv M_{\mathrm{env}, 0} / M_{\mathrm{sc}}=4.0,3.1$, and 2.1 , respectively, on the $0.98 M_{\odot}$ WD model. The dotted line represents an approximate relation of Equation (12).

We tabulate $M_{\mathrm{sc}}$ for each $M_{\mathrm{WD}}$ in Tables 1 (CO novae), 2 (Ne novae), and 3 (Solar abundance). Then, the 1mag, 2-mag, and 3-mag decays from the peak are defined along this approximate line, that is,

$$
\begin{aligned}
& 1=9.5 \log \left(\frac{M_{\mathrm{env}, 0}}{M_{\mathrm{env}, 1}}\right), \\
& 2=9.5 \log \left(\frac{M_{\mathrm{env}, 0}}{M_{\mathrm{env}, 2}}\right),
\end{aligned}
$$

and

$$
3=9.5 \log \left(\frac{M_{\mathrm{env}, 0}}{M_{\mathrm{env}, 3}}\right),
$$

where $M_{\mathrm{env}, 0}$ is the envelope mass at the peak, $M_{\mathrm{env}, 1}$ at the 1-mag decay, $M_{\mathrm{env}, 2}$ at the 2-mag decay, and $M_{\mathrm{env}, 3}$ at the 3 -mag decay from the peak. We regard $M_{\mathrm{env}, 0}$ to be the same as the ignition mass. We derive

$$
\begin{aligned}
& M_{\mathrm{env}, 1}=0.785 M_{\mathrm{env}, 0}, \\
& M_{\mathrm{env}, 2}=0.616 M_{\mathrm{env}, 0},
\end{aligned}
$$

and

$$
M_{\mathrm{env}, 3}=0.483 M_{\mathrm{env}, 0} .
$$

This means that the brightness drops by 1, 2, and 3 mag when the envelope mass is lost in the wind and decreases to $0.785,0.616$, and 0.483 times the initial envelope mass, respectively. This property is independent of the WD mass or chemical composition. Thus, we use the envelope mass that characterizes the evolution of a nova outburst instead of the time since the optical/IR maximum.

Using the above property, we can rewrite the $t_{1}, t_{2}$, and $t_{3}$ times as a function of the envelope mass. Here, $t_{1}, t_{2}$, and $t_{3}$ times are

$$
\begin{aligned}
& t_{1}=\int_{M_{\mathrm{env}, 0}}^{M_{\mathrm{env}, 1}} \frac{d M_{\mathrm{env}}}{\dot{M}_{\mathrm{env}}}, \\
& t_{2}=\int_{M_{\mathrm{env}, 0}}^{M_{\mathrm{env}, 2}} \frac{d M_{\mathrm{env}}}{\dot{M}_{\mathrm{env}}},
\end{aligned}
$$

and

$$
t_{3}=\int_{M_{\mathrm{env}, 0}}^{M_{\mathrm{env}, 3}} \frac{d M_{\mathrm{env}}}{\dot{M}_{\mathrm{env}}} .
$$

We analytically approximate the wind mass loss rate by the dotted line in Figure 3(b), that is,

$$
\log \left(\frac{-\dot{M}_{\mathrm{env}}}{M_{\odot} \mathrm{yr}^{-1}}\right)=2.5 \log \left(\frac{M_{\mathrm{env}}}{M_{\mathrm{sc}}}\right)-4.7 .
$$

This equation can be rewritten as

$$
-\dot{M}_{\mathrm{env}}=f_{\mathrm{s}} C_{\mathrm{wind}}\left(\frac{M_{\mathrm{env}}}{M_{\mathrm{sc}}}\right)^{2.5},
$$


Table 2. Scaling Masses for Neon Novae ${ }^{a}$

\begin{tabular}{|c|c|c|}
\hline $\begin{array}{l}M_{\mathrm{WD}} \\
\left(M_{\odot}\right)\end{array}$ & $\begin{array}{c}M_{\mathrm{Sc}}(\mathrm{Ne} 2) \\
\left(10^{-5} M_{\odot}\right)\end{array}$ & $\begin{array}{c}M_{\mathrm{sc}}(\mathrm{Ne} 3) \\
\left(10^{-5} M_{\odot}\right)\end{array}$ \\
\hline 0.70 & 2.10 & 2.73 \\
\hline 0.75 & 1.64 & 2.13 \\
\hline 0.80 & 1.30 & 1.68 \\
\hline 0.85 & 1.03 & 1.33 \\
\hline 0.90 & 0.823 & 1.06 \\
\hline 0.95 & 0.685 & 0.876 \\
\hline 1.00 & 0.568 & 0.724 \\
\hline 1.05 & 0.448 & 0.568 \\
\hline 1.10 & 0.353 & 0.447 \\
\hline 1.15 & 0.280 & 0.353 \\
\hline 1.20 & 0.223 & 0.280 \\
\hline 1.25 & 0.163 & 0.204 \\
\hline 1.30 & 0.113 & 0.141 \\
\hline 1.33 & 0.0810 & 0.100 \\
\hline 1.35 & 0.0603 & 0.0743 \\
\hline \multicolumn{3}{|c|}{$\begin{array}{l}a_{\text {chemical composition of the }} \text { of the } \\
\text { hydrogen-rich envelope is as- } \\
\text { sumed to be those of "Ne } \\
\text { nova 2" and "Ne nova } 3 " \text { in } \\
\text { Table } 2 \text { of Hachisu \& Kato } \\
(2006), \text { i.e., }\left(X, Y, Z, X_{\mathrm{O}}, X_{\mathrm{Ne}}\right)= \\
(0.55,0.30,0.02,0.10,0.03) \text { and } \\
(0.55,0.37,0.02,0.03,0.03), \text { respec- } \\
\text { tively. }\end{array}$} \\
\hline
\end{tabular}

together with the proportionality constant of

$$
C_{\text {wind }}=10^{-4.7} M_{\odot} \mathrm{yr}^{-1} .
$$

Using Equation (23) together with the $x$-parameter, $x \equiv$ $M_{\mathrm{env}} / M_{\mathrm{sc}}$, we derive the elapsed time from the optical peak, i.e.,

$$
t=\frac{M_{\mathrm{sc}}}{C_{\mathrm{wind}}} \int_{x}^{x_{0}} \frac{d x}{x^{2.5}}=\frac{M_{\mathrm{sc}}}{1.5 C_{\mathrm{wind}}}\left(x^{-1.5}-x_{0}^{-1.5}\right),
$$

and

$$
\tau \equiv t / f_{\mathrm{s}}=54.7\left(x^{-1.5}-x_{0}^{-1.5}\right) \text { days. }
$$

Then, the $t_{1}$ time is calculated from

$$
\begin{aligned}
t_{1} & =\frac{M_{\mathrm{sc}}}{C_{\text {wind }}} \int_{x_{1}}^{x_{0}} \frac{d x}{x^{2.5}}=\frac{M_{\mathrm{sc}}}{1.5 C_{\text {wind }}}\left(x_{1}^{-1.5}-x_{0}^{-1.5}\right) \\
& =\frac{M_{\mathrm{sc}}}{1.5 f_{\mathrm{s}} C_{\text {wind }} x_{0}^{1.5}}\left(0.785^{-1.5}-1\right) \\
& =\frac{0.438 M_{\mathrm{sc}}}{1.5 C_{\text {wind }} x_{0}^{1.5}},
\end{aligned}
$$

\begin{tabular}{|c|c|}
\hline $\begin{array}{l}M_{\mathrm{WD}} \\
\left(M_{\odot}\right)\end{array}$ & $\begin{array}{c}M_{\mathrm{Sc}} \\
\left(10^{-5} M_{\odot}\right)\end{array}$ \\
\hline 0.55 & 7.07 \\
\hline 0.60 & 5.88 \\
\hline 0.65 & 4.29 \\
\hline 0.70 & 3.35 \\
\hline 0.75 & 2.66 \\
\hline 0.80 & 2.22 \\
\hline 0.85 & 1.76 \\
\hline 0.90 & 1.39 \\
\hline 0.95 & 1.15 \\
\hline 1.00 & 0.948 \\
\hline 1.05 & 0.739 \\
\hline 1.10 & 0.581 \\
\hline 1.15 & 0.456 \\
\hline 1.20 & 0.360 \\
\hline 1.25 & 0.261 \\
\hline 1.30 & 0.180 \\
\hline 1.33 & 0.127 \\
\hline 1.35 & 0.0940 \\
\hline \multicolumn{2}{|c|}{$\begin{array}{l}\text { a chemical composition } \\
\text { of the envelope is } \\
\text { assumed to be that } \\
\text { "Solar" in Table } 2 \\
\text { Hachisu \& Kato } \\
(2006), \\
(X, Y, Z) \\
(0.70,0.28,0.02) .\end{array}$} \\
\hline
\end{tabular}

and the $t_{2}$ time is

$$
t_{2}=\frac{M_{\mathrm{sc}}}{1.5 f_{\mathrm{s}} C_{\mathrm{wind}} x_{0}^{1.5}}\left(0.616^{-1.5}-1\right)
$$

Table 3. Scaling Masses for Solar abundance $^{a}$

$$
=\frac{1.068 M_{\mathrm{sc}}}{1.5 C_{\text {wind }} x_{0}^{1.5}},
$$

and the $t_{3}$ time is

$$
\begin{aligned}
t_{3} & =\frac{M_{\mathrm{sc}}}{1.5 C_{\text {wind }} x_{0}^{1.5}}\left(0.483^{-1.5}-1\right) \\
& =\frac{1.979 M_{\mathrm{sc}}}{1.5 C_{\text {wind }} x_{0}^{1.5}} .
\end{aligned}
$$

From Equations (27) and (28), we have a simple relation between $t_{1}$ and $t_{2}$ as

$$
t_{1}=0.41 t_{2},
$$

and, from Equations (28) and (29), we have

$$
t_{2}=0.54 t_{3} .
$$

It should be noted that Equations (22) and (23) are approximately valid for $\log \left(-\dot{M}_{\text {env }} / M_{\odot} \mathrm{yr}^{-1}\right) \gtrsim-4.8$ because each line begins to diverge below that rate as 
shown in Figure 3(b). Therefore, our estimates by Equations (27), (28), and (29) are approximately valid for $x_{0} \gtrsim 1.16, x_{0} \gtrsim 1.5$, and $x_{0} \gtrsim 1.9$, respectively. These lower bounds correspond to $\log \left(-\dot{M}_{\text {env }} / M_{\odot} \mathrm{yr}^{-1}\right)=$ -4.8 at $M_{\mathrm{env}, 1}, M_{\mathrm{env}, 2}$ and $M_{\mathrm{env}, 3}$, respectively.

We compare our approximate relations of Equations (28) and (29) with the V1668 Cyg light curve. If we adopt a $0.98 M_{\odot}$ WD (CO3) model, we have $x_{0}=3.1$ from Figure 4 , and $M_{\mathrm{sc}}=0.448 \times 10^{-5} M_{\odot}$ from Table 1. Then, we obtain $t_{2}=10.7$ days, $t_{3}=19.8$ days, and the ratio $t_{2} / t_{3}=10.7 / 19.8=0.54$. These $t_{2}$ and $t_{3}$ values are slightly shorter than, but approximately consistent with, the observation, e.g., $t_{2}=12.2$ days and $t_{3}=24.3$ days (for $V$ band) in Mallama \& Skillman (1979), or $t_{2}=12$ days and $t_{3}=23$ days (for $V$ band) in di Paolantonio et al. (1981).

Hachisu \& Kato (2006) discussed the relation between $t_{2}$ and $t_{3}$ based on the universal decline law because it has a slope of $F_{\nu} \propto t^{-1.75}$. They obtained the relation $t_{3}=1.69 t_{2}+0.69 \Delta t_{0}$, where $\Delta t_{0}$ is the time from the outburst to optical maximum. Usually $\Delta t_{0}$ is short compared with $t_{2}$ and $t_{3}$. Then, we have $t_{2}=(1 / 1.69) t_{3}=0.59 t_{3}$. This is approximately equivalent to the result of Equation (31). Hachisu \& Kato (2006) compared their results with the observation. For example, Capaccioli et al. (1990) obtained $t_{3}=$ $(1.68 \pm 0.08) t_{2}+(1.9 \pm 1.5)$ days for $t_{3}<80$ days, or $t_{3}=(1.68 \pm 0.04) t_{2}+(2.3 \pm 1.6)$ days for $t_{3}>80$ days. If $t_{3} \gg 3$ days, we obtain $t_{2}=(1 / 1.68) t_{3}=0.60 t_{3}$. This is also approximately equivalent to Equation (31).

Recent work done by Özdörmez et al. (2018) concluded, however, that the relation between $t_{2}$ and $t_{3}$ is not unique but different among various types of nova light curve shapes defined by Strope et al. (2010). Özdörmez et al. (2018) obtained $\log t_{3}=0.96 \log t_{2}+$ 0.32 for $\mathrm{S}$ (smooth) -type, $\log t_{3}=0.92 \log t_{2}+0.43$ for $\mathrm{P}$ (plateau) -type, $\log t_{3}=0.72 \log t_{2}+0.6$ for $\mathrm{D}$ (dip) -type, and $\log t_{3}=0.46 \log t_{2}+1.29$ for $\mathrm{J}$ (jitter) -type. The S-type relation corresponds to $t_{2} \approx 0.5\left(t_{3}\right)^{1.04}$. This is consistent with Equation (31). We should note that the physical meaning of $t_{2}$ or $t_{3}$ is a local decline trend near the optical peak. If a light curve has multiple peaks, secondary maximum, oscillations, early dust blackout, jitters, or flares, we should not apply $t_{2}$ or $t_{3}$ because $t_{2}$ or $t_{3}$ is greatly affected by such local variations.

Not all but rather many novae broadly follow the universal decline law (e.g., Hachisu \& Kato 2006, 2007, 2010, 2016, 2018, 2019a,b). Strictly speaking, the universal decline law is well applied to S-type light curve shape novae defined by Strope et al. (2010). Such an example is V1668 Cyg in Figure 1. The other types of nova light curve shapes deviate from our model light curves in some part. However, their global trends of decline can be sometimes fitted with our model light curves. Our approximate formulae mentioned above are valid for such novae.

\section{THEORETICAL MMRD RELATION}

\subsection{The MMRD Relation of the Universal Decline Law}

In the previous section, we formulated the nova model light curves for various WD masses and chemical compositions by the two parameters of $\left(M_{\mathrm{sc}}, x_{0}\right)$. In this section, we convert these two parameters to $\left(t_{3}, M_{V \text {, max }}\right)$. From Equation (29), we have

$$
t_{3}=19.8\left(\frac{M_{\mathrm{sc}}}{4.48 \times 10^{-6} M_{\odot}}\right)\left(\frac{x_{0}}{3.1}\right)^{-1.5} \text { days. }
$$

This is approximately valid for $x_{0} \gtrsim 2.0$ as noted in Section 3. Also from Equation (12), we have

$$
\begin{aligned}
M_{V, \max }= & 2.5 \log \left(\frac{M_{\mathrm{sc}}}{4.48 \times 10^{-6} M_{\odot}}\right) \\
& -9.5 \log \left(\frac{x_{0}}{3.1}\right)-8.4 .
\end{aligned}
$$

This is approximately valid for $x_{0} \gtrsim 1.0$ as shown in Figure 4. To summarize, we can apply Equations (32) and (33) for $x_{0} \gtrsim 2.0$.

We plot equi- $M_{\mathrm{sc}}$ lines and equi- $x_{0}$ lines in Figure 5. The solid magenta lines represent each equi- $M_{\mathrm{sc}}$ line corresponding to the $M_{\mathrm{sc}}$ values in Table 3. The thin solid blue lines denote each equi- $x_{0}$ line, from bottom to top, $x_{0}=1,1.5,2,2.5,3,3.5,4,5,6$, and 7 . The thick yellow line corresponds to the $x_{0}=2$ line, above which Equations (32) and (33) are approximately valid.

We add observational points taken from Downes \& Duerbeck (2000) with filled red circles and Selvelli \& Gilmozzi (2019) with filled red stars. We also add an unfilled red star at the position of V1500 Cyg taken from della Valle \& Izzo (2020). For the distance to a nova, Downes \& Duerbeck (2000) used the expansion parallax method of nova shells while Selvelli \& Gilmozzi (2019) and della Valle \& Izzo (2020) used the trigonometric parallaxes of Gaia Data Release 2 (Gaia DR2). These two data show a similar trend in the $\left(\log t_{3}\right)-M_{V, \max }$ diagram. We also add two linear trend lines of $M_{V, \max }=-11.08+2.12 \log t_{3}$ (thick solid black line) and $M_{V, \max }=-11.99+2.54 \log t_{3}$ (thick solid cyan line) that represent linear trends derived by Selvelli \& Gilmozzi (2019) and Downes \& Duerbeck (2000), respectively. The upper-left outlined red star is for GK Per while the lower-right outlined red star is for V533 Her both from Selvelli \& Gilmozzi (2019). We discuss these two novae in Section 5. 


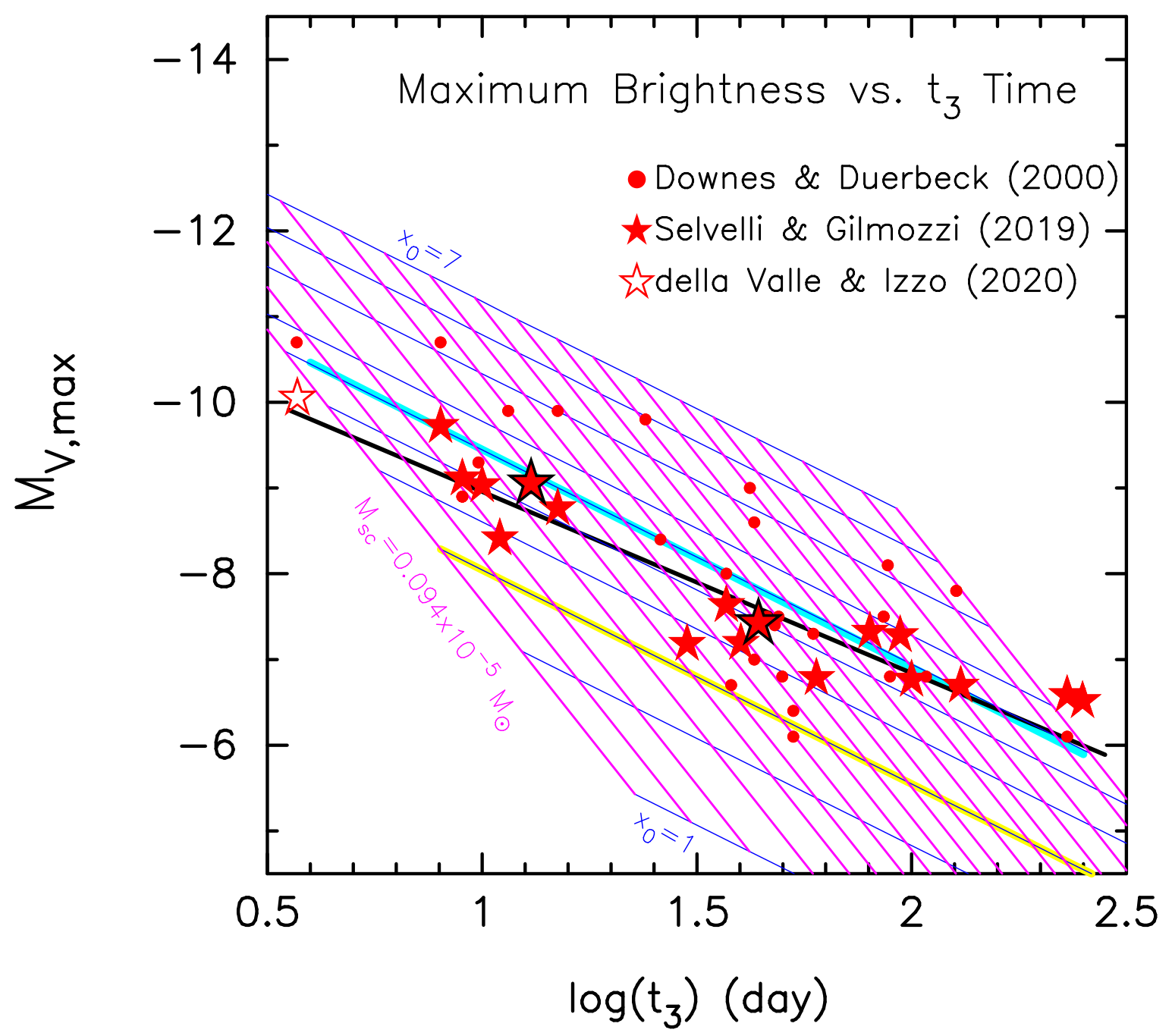

Figure 5. The maximum $V$ magnitude $M_{V \text {, max }}$ against the rate of decline $t_{3}$ for various equi- $M_{\mathrm{sc}}$ (solid magenta lines) and equi- $x_{0}$ (solid blue lines) models. The $M_{V \text {,max }}$ and $t_{3}$ are calculated from Equations (33) and (32), respectively. The filled red circles and stars are for galactic novae obtained by Downes \& Duerbeck (2000) and Selvelli \& Gilmozzi (2019), respectively. We also add an unfilled red star at the position of V1500 Cyg taken from della Valle \& Izzo (2020). The upper-left outlined red star is for GK Per while the lower-right outlined red star is for V533 Her. The thick solid cyan line represents the "classical" MMRD relation defined by Downes \& Duerbeck (2000), i.e., $M_{V \text {, max }}=-11.99+2.54 \log \left(t_{3}\right)$ while the thick solid black line represents the linear MMRD relation defined by Selvelli \& Gilmozzi (2019), i.e., $M_{V, \max }=-11.08+2.12 \log \left(t_{3}\right)$. The solid magenta lines connect the same scaling mass $M_{\mathrm{sc}}$ but for different $x_{0}$. The scaling masses are, from right to left, the same as those tabulated in Table 3. The thin solid blue lines connect the same $x_{0}$, i.e., $x_{0}=1,1.5,2,2.5,3,3.5,4,5,6$, and 7 , from lower to upper. The thick yellow line corresponds to the $x_{0}=2$ line, above which Equation (32) is approximately valid. The magenta lines of equi- $M_{\mathrm{sc}}$ have a slope of 6.3 while the blue lines of equi- $x_{0}$ have a slope of 2.5 in the (log $\left.t_{3}\right)-M_{V, \text { max }}$ diagram.

The equi- $M_{\mathrm{sc}}$ lines have a slope of $6.3(=9.5 / 1.5)$ while the equi- $x_{0}$ lines have a slope of 2.5 in the $\left(\log t_{3}\right)$ $M_{V, \max }$ diagram. The latter slope is close to 2.54 of the cyan line, the trend MMRD line defined by Downes \& Duerbeck (2000), but slightly steeper than the slope of 2.12 (thick solid black line) obtained by Selvelli \& Gilmozzi (2019). This thick black line traverses the two blue lines of $x_{0}=3.0$ and 3.5 from left to right. The observational MMRD points (both the filled red circles and stars) are covered with the region between the lower bound $x_{0} \sim 2$ and the up- per bound $x_{0} \sim 6$, the center of which is $x_{0} \sim 3.5$. Hachisu \& Kato (2010) examined the observational MMRD distribution obtained by Downes \& Duerbeck (2000, filled red circles) and reached a similar conclusion (see Figure 15 of Hachisu \& Kato 2010). This is because the trend MMRD line (cyan line) obtained by Downes \& Duerbeck (2000) almost overlaps with the blue $x_{0}=3.5$ line.

It should be noted that the global timescale of a nova light curve is $f_{\mathrm{s}} \propto M_{\mathrm{sc}}$ as shown in Equations (10) and (11). On the other hand, the $t_{3}$ time is not a global 
timescale but a local timescale only near the peak of a nova light curve. The $t_{3}$ time is proportional to $f_{\mathrm{s}}(\propto$ $M_{\text {sc }}$ ) but depends also on the $x_{0}$ as in Equation (29) or (32). This is the reason why the MMRD points show a large scatter around the main trend in the $\left(\log t_{3}\right)$ $M_{V \text {, max }}$ diagram. This can be easily understood if we eliminate $M_{\text {sc }}$ from Equations (32) and (33) and obtain

$$
M_{V, \max }=2.5 \log t_{3}-11.95-5.75 \log \left(\frac{x_{0}}{3.5}\right) .
$$

Then, we have

$$
M_{V, \max }=2.5 \log t_{3}-11.95, \text { for } x_{0}=3.5 .
$$

This MMRD relation is essentially the same as that (cyan line) obtained by Downes \& Duerbeck (2000), but slightly steeper than the trend MMRD line (black line) obtained by Selvelli \& Gilmozzi (2019) in Figure 5 . The scatter from the main trend $\left(x_{0} \sim 3.0-3.5\right.$ line $)$ can be understood from the difference in $x_{0}$, that is, the difference in the ignition mass.

\subsection{The MMRD Relation for Novae}

In this subsection, we obtain $\left(t_{3}, M_{V \text {, max }}\right)$ against nova models, which are specified by the mass accretion rate $\dot{M}_{\text {acc }}$ and the WD mass $M_{\mathrm{WD}}$ (or sometimes by the recurrence time $\left.t_{\text {rec }}\right)$. These parameters are determined by the binary nature. We adopt the ignition mass $M_{\text {ig }}$, mass accretion rate $\dot{M}_{\text {acc }}$, and recurrence time $t_{\text {rec }}$ from published data available to the authors.

\subsubsection{Ignition mass model}

The ignition masses have been calculated by many authors (e.g., Prialnik \& Kovetz 1995; Kato et al. 2014; Hachisu et al. 2016; Chen et al. 2019). However, the ignition masses can sometimes differ significantly each other, depending not only on the model assumption but also on the calculation method (numerical code, see, e.g., Kato et al. 2017a).

We adopt the result of Kato et al. (2014). They calculated the accumulation mass $M_{\text {acc }}$ and ignition mass $M_{\text {ig }}$ assuming solar abundance. They obtained models for $\dot{M}_{\text {acc }} \geq 1 \times 10^{-9} M_{\odot} \mathrm{yr}^{-1}$. In the present paper, we extend the mass accretion rate down to $\dot{M}_{\text {acc }} \geq 1 \times 10^{-11} M_{\odot} \mathrm{yr}^{-1}$ taking into account the lower mass-accretion rate limit for cataclysmic variables (e.g., Knigge et al. 2011). We tabulate $M_{\text {acc }}$ (third column) in Table 4 for various WD masses $M_{\mathrm{WD}}$ (first column) and mass accretion rates $\dot{M}_{\text {acc }}$ (second column). We assume that the envelope mass at optical maximum $M_{\text {env, } 0}$ is almost the same as the ignition mass $M_{\text {ig }}$, i.e., $M_{\text {env }, 0}=M_{\text {ig }}$. Using the scaling mass $M_{\mathrm{sc}}$ in Table 3 , we calculate the ratio of $x_{0} \equiv M_{\mathrm{env}, 0} / M_{\mathrm{sc}}=M_{\mathrm{ig}} / M_{\mathrm{sc}}$.
These $x_{0}$ are also tabulated on the fifth column in Table 4.

It should be noted that the accumulation mass $M_{\text {acc }}=$ $t_{\text {rec }} \times \dot{M}_{\text {acc }}$ is slightly smaller than the envelope mass at ignition $M_{\mathrm{ig}}=M_{\mathrm{env}, 0}=x_{0} \times M_{\mathrm{sc}}$. When the mass accretion starts, there is residual hydrogen-rich material on the WD. This residual is leftover from the previous nova explosion. The ignition mass is defined by the summation of the accreted mass and the envelope mass at the epoch when hydrogen burning extinguishes, i.e.,

$$
M_{\mathrm{ig}}=M_{\mathrm{env}, 0}=M_{\mathrm{acc}}+M_{\mathrm{env}, \min }
$$

(See Figure 1 of Kato et al. (2014) for the relation among these envelope masses.)

For a given ignition mass, we obtain the absolute $V$ magnitude at optical maximum $M_{V \text {, max }}$ from Equation (33). The values of $M_{V \text {, max }}$ are tabulated at 6 th column in Table 4. We obtain the $t_{3}$ time from Equation (32) and $t_{2}$ time from Equation (31). These are also tabulated in Table 4.

\subsubsection{Global trend of MMRD relation}

We plot these peak $V$ brightness versus rate of decline relation in Figure 6. Each thin solid blue line connects the same WD mass models with different mass accretion rates. The thick solid gray lines connect the models with the same mass accretion rate. These gray lines have a peak of $M_{V \text {, max }}$ at $M_{\mathrm{WD}}=1.1 M_{\odot}$. The thin red lines connect the same recurrence period models.

In this figure, we add the observational MMRD points, filled red circles, filled red stars, and an unfilled red star, obtained by Downes \& Duerbeck (2000), Selvelli \& Gilmozzi (2019), and della Valle \& Izzo (2020), respectively. We can see that the distribution of MMRD points is covered by mass accretion rates between $\dot{M}_{\text {acc }} \sim 1 \times 10^{-11} M_{\odot} \mathrm{yr}^{-1}$ and $\dot{M}_{\text {acc }} \sim$ $3 \times 10^{-8} M_{\odot} \mathrm{yr}^{-1}$ and centered on $\dot{M}_{\text {acc }} \sim 5 \times$ $10^{-9} M_{\odot} \mathrm{yr}^{-1}$ in the longer $t_{3}$ region of $t_{3} \gtrsim 30$ days, based on the result of Kato et al. (2014).

The thick solid black line is a trend of MMRD distribution obtained by Selvelli \& Gilmozzi (2019), which is close to an equi-mass accretion rate line of $\dot{M}_{\text {acc }} \sim$ $5 \times 10^{-9} M_{\odot} \mathrm{yr}^{-1}$ in the longer $t_{3}$ region of $t_{3} \gtrsim 30$ days (or in the fainter region of $M_{V, \max } \geq-8.0$ ).

On the other hand, the thick black line traverses the four gray lines of $\dot{M}_{\text {acc }}=3 \times 10^{-9} M_{\odot} \mathrm{yr}^{-1}$, $1 \times 10^{-9} M_{\odot} \mathrm{yr}^{-1}, 1 \times 10^{-10} M_{\odot} \mathrm{yr}^{-1}$, and $1 \times$ $10^{-11} M_{\odot} \mathrm{yr}^{-1}$ in the brighter region of $M_{V, \max } \leq$ -8.0. If we divide the data set (filled red stars) of Selvelli \& Gilmozzi (2019) into two groups by the brightness of $M_{V \text {, max }}=-8.0$, the brighter (upperleft) group is located in between $\dot{M}_{\text {acc }}=1 \times 10^{-9}$ 


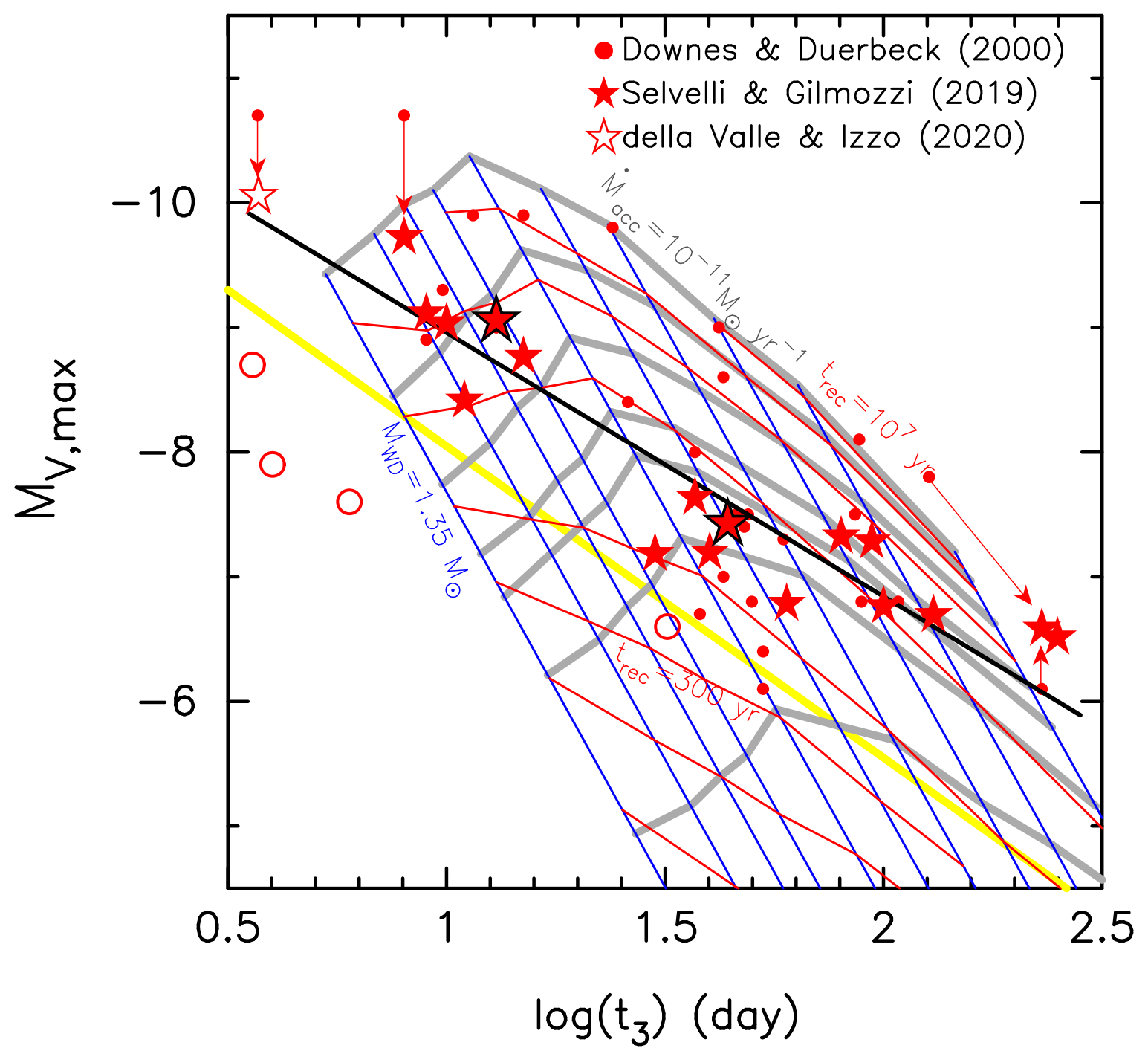

Figure 6. Same as Figure 5 , but for equi- $M_{\mathrm{WD}}$, equi- $\dot{M}_{\mathrm{acc}}$, and equi- $t_{\mathrm{rec}}$ models of solar abundance. The thin solid blue lines connect the same WD mass $M_{\mathrm{WD}}$ but for different mass accretion rates $\dot{M}_{\text {acc }}$. The WD masses are, from right to left, $M_{\mathrm{WD}}=0.6,0.7,0.8,0.9,1.0,1.1,1.2,1.25,1.3$, and $1.35 M_{\odot}$. The thick yellow line corresponds to the $x_{0}=2$ line. The thin solid red lines connect the same recurrence time, i.e., $t_{\mathrm{rec}}=30,100,300,1000,10000,10^{5}, 10^{6}$, and $10^{7} \mathrm{yr}$, from lower to upper. The thick solid gray lines represent the same mass accretion rate, from lower to upper, $\dot{M}_{\text {acc }}=3 \times 10^{-8}, 1 \times 10^{-8}, 5 \times 10^{-9}$, $3 \times 10^{-9}, 1 \times 10^{-9}, 1 \times 10^{-10}$, and $1 \times 10^{-11} M_{\odot} \mathrm{yr}^{-1}$. See Table 4 for each data. The four unfilled red circles denote the MMRD positions of the four recurrent novae, CI Aql, T CrB, U Sco, and V745 Sco. Other symbols and lines are the same as those in Figure 5. See text for more details.

and $1 \times 10^{-11} M_{\odot} \mathrm{yr}^{-1}$ and the fainter (lower-right) group is located in between $\dot{M}_{\text {acc }}=3 \times 10^{-8}$, and $3 \times 10^{-9} M_{\odot} \mathrm{yr}^{-1}$. This difference in the mass accretion rate broadly correspond to cataclysmic variables below/above the period gap (e.g., Knigge et al. 2011).

For an MMRD point of the observed nova, we are able to broadly specify the WD mass and mass accretion rate. The main trend in the MMRD diagram corresponds to the mass accretion rate of $\dot{M}_{\text {acc }} \sim 5 \times 10^{-9} M_{\odot} \mathrm{yr}^{-1}$ with the different WD masses in the lower region of $M_{V, \max } \geq$ -8.0. Selvelli \& Gilmozzi (2019) estimated the mass accretion rates from the quiescent luminosities of their data set novae in Figure 6 (filled red stars). Their median value is $\dot{M}_{\mathrm{WD}}=3.3 \times 10^{-9} M_{\odot} \mathrm{yr}^{-1}$, which is close to our main trend value of $\dot{M}_{\text {acc }} \sim 5 \times 10^{-9} M_{\odot} \mathrm{yr}^{-1}$. The scatter up to $\pm 3 \sigma= \pm 1.0 \mathrm{mag}$ from the main trend line can be attributed to the different mass accretion rates. Thus, the global trend of an MMRD relation does indeed exist, at least, in the fainter region of $M_{V, \max } \geq-8.0$, but its scatter is too large for it to be a precision distance indicator of individual novae.

Selvelli \& Gilmozzi (2019) reported a correlation between $\dot{M}_{\text {acc }}$ and the speed class $t_{3}\left(\dot{M}_{\text {acc }}\right.$ increasing with $t_{3}$ as in their Figure 5) for their 17 nova data set and 
wrote "we cannot find a simple explanation that could account for this." This correlation $\left(\dot{M}_{\text {acc }}\right.$ increases with $t_{3}$ ) can be easily seen up to $t_{3} \lesssim 80$ days for the filled red stars in our Figure 6. For $t_{3} \gtrsim 80$ days, this correlation seems to be flat except for RR Pic and HR Del. This global trend of our $\dot{M}_{\text {acc }}$ versus $t_{3}$ relation is consistent with their Figure 5.

\subsubsection{MMRD positions of individual novae}

Finally, we point out that there is a -10.4 mag cap for the maximum $V$ brightness if we limit the mass accretion rate above $\dot{M}_{\text {acc }} \geq 1 \times 10^{-11} M_{\odot} \mathrm{yr}^{-1}$.

Several novae are located outside our region of $\left(t_{3}, M_{V, \max }\right)$. They are, from left to right, V1500 Cyg (red filled circle: $t_{3}=3.7$ days, $M_{V, \max }=-10.7$ ), CP Pup (red filled circle: 8 days, -10.7), RR Pic (red filled circles: 127 days, -7.8), HR Del (red filled circle: 230 days, -6.1 ). The data of V1500 Cyg is revised by della Valle \& Izzo (2020) to be $M_{V, \max }=-10.05$ (unfilled red star). The data of CP Pup, RR Pic, and HR Del are revised by Selvelli \& Gilmozzi (2019) to be $M_{V, \max }=-9.72$ and $t_{3}=8$ days (CP Pup, filled red star), -6.51 and 250 days (RR Pic, filled red star), and -6.58 and 230 days (HR Del, filled red star). These four corrections are indicated by the red arrows in Figure 6 .

This explains that the brightest peaks of novae are fainter than $M_{V} \sim-10.4$ (e.g., Cohen 1985; Shafter et al. 2011; Cao et al. 2012; Shafter 2013; Shara et al. 2017; della Valle \& Izzo 2020). The brightness cap $\left(M_{V} \sim-10.4\right)$ of classical novae is constrained by the lowest mass-accretion rate of $\dot{M}_{\text {acc }} \sim$ $1 \times 10^{-11} M_{\odot} \mathrm{yr}^{-1}$.

In the longer $t_{3}$ time, only two novae (HR Del and RR Pic) are outside our theoretical region between $M_{\mathrm{WD}}=0.6$ and $1.35 M_{\odot}$ and between $\dot{M}_{\mathrm{acc}}=3 \times$ $10^{-8} M_{\odot} \mathrm{yr}^{-1}$ and $1 \times 10^{-11} M_{\odot} \mathrm{yr}^{-1}$. This simply means (1) that the WD mass is smaller than 0.6 $M_{\odot}$ or (2) that the concept of $t_{2}$ and $t_{3}$ time should not be applied to these novae for some reasons. These two novae have multiple peaks (see, e.g., Figure 1 of Hachisu \& Kato 2015). The $t_{2}$ or $t_{3}$ time is a local timescale near the peak and is greatly affected by such variations. We should not apply $t_{2}$ and $t_{3}$ to such novae. Hachisu \& Kato (2015) analyzed the light curves of HR Del and RR Pic and globally fitted their model light curves of $M_{\mathrm{WD}}=0.55 M_{\odot}$ and $0.51 M_{\odot}$ with the observed light curves of these two novae except the multiple peaks.

GK Per and V446 Her belong to the shortest $t_{3}$ and brightest $M_{V, \max }$ group of Selvelli \& Gilmozzi's nova data set, i.e., $\left(t_{3}, M_{V, \max }\right)=(13,-9.05)$ and $(15,-8.76)$. These two novae show dwarf nova outbursts in a post- nova phase, suggesting that their mass accretion rates are now quite low, below a few times $10^{-9} M_{\odot} \mathrm{yr}^{-1}$ (e.g., Knigge et al. 2011). Figure 6 indicates relatively low mass accretion rates of $\sim 2 \times 10^{-10}$ and $\sim 7 \times 10^{-10} M_{\odot} \mathrm{yr}^{-1}$. Selvelli \& Gilmozzi (2019) estimated the mass accretion rates, $\dot{M}_{\text {acc }}=2 \times 10^{-9}$ and $0.8 \times 10^{-9} M_{\odot} \mathrm{yr}^{-1}$, respectively. Their estimated rate of V446 Her seems to be consistent with our theoretical value estimated from the position in the $\left(\log t_{3}\right)-M_{V, \max }$ diagram while that of GK Per is about ten times larger than our value. This suggests that, for a certain kind of novae, the mass accretion rate during a post-nova phase is rather high compared with that of a substantial prenova phase, or that the mass accretion rate gradually decreases toward much lower rates in a timescale of recurrence period.

CP Pup has a lowest value of $\dot{M}_{\text {acc }}$ in Selvelli \& Gilmozzi's nova data set, i.e., $\dot{M}_{\text {acc }}=0.6 \times$ $10^{-9} M_{\odot} \mathrm{yr}^{-1}$. On the other hand, Figure 6 indicates $\dot{M}_{\text {acc }}=(1.5-2) \times 10^{-11} M_{\odot} \mathrm{yr}^{-1}$. This is about 30 times smaller than that of Selvelli \& Gilmozzi. Schaefer \& Collazzi (2010) reported that the pre-nova brightness of CP Pup is $\Delta m \sim 5$ mag fainter than that of a post-nova brightness. This simply indicates that the mass-accretion rate is $\sim 100$ times smaller than that during a post-nova phase. If the $\dot{M}_{\text {acc }}$ decreases toward $\dot{M}_{\text {acc }}=0.6 \times 10^{-11} M_{\odot} \mathrm{yr}^{-1}$ at the pre-nova phase, the average mass-accretion rate during the quiescent phase is close to $\dot{M}_{\text {acc }} \sim 1 \times 10^{-11} M_{\odot} \mathrm{yr}^{-1}$. This average value is consistent with our theoretical estimate.

\subsubsection{MMRD positions of recurrent novae}

Recurrent novae (unfilled red circles) are located below the yellow line in Figure 6. Here, we plot four MMRD positions of the recurrent novae, CI Aql $\left(t_{3}=\right.$ 32 days, $\left.M_{V, \max }=-6.6\right)$, $\mathrm{T} \mathrm{CrB}(6,-7.6)$, U Sco $(3.6,-8.7)$, and V745 Sco $(4,-7.9)$. The data are taken from Table 2 of Hachisu \& Kato (2018) except for CI Aql. The data of CI Aql are calculated from $m_{V, \max }=9.0$ (Strope et al. 2010), $A_{V}=3.1 E(B-$ $V)=3.1 \times 1.0=3.1$ (Hachisu \& Kato 2018), and $d=3189_{-315}^{+949} \mathrm{pc}$ (Gaia distance, Schaefer 2018). We exclude RS Oph and T Pyx. This is because the light curve of RS Oph is contaminated by the shock-heating between the ejecta and circumstellar matter and, as a result, the $t_{3}$ time does not represent the timescale of intrinsic decline (e.g., Hachisu \& Kato 2018). T Pyx has multiple peaks and we should not apply the concept of $t_{3}$.

The recurrence periods of these recurrent novae are $\sim 10-80$ yr (e.g., Schaefer 2010). It is obvious that the MMRD positions of these recurrent novae are not con- 

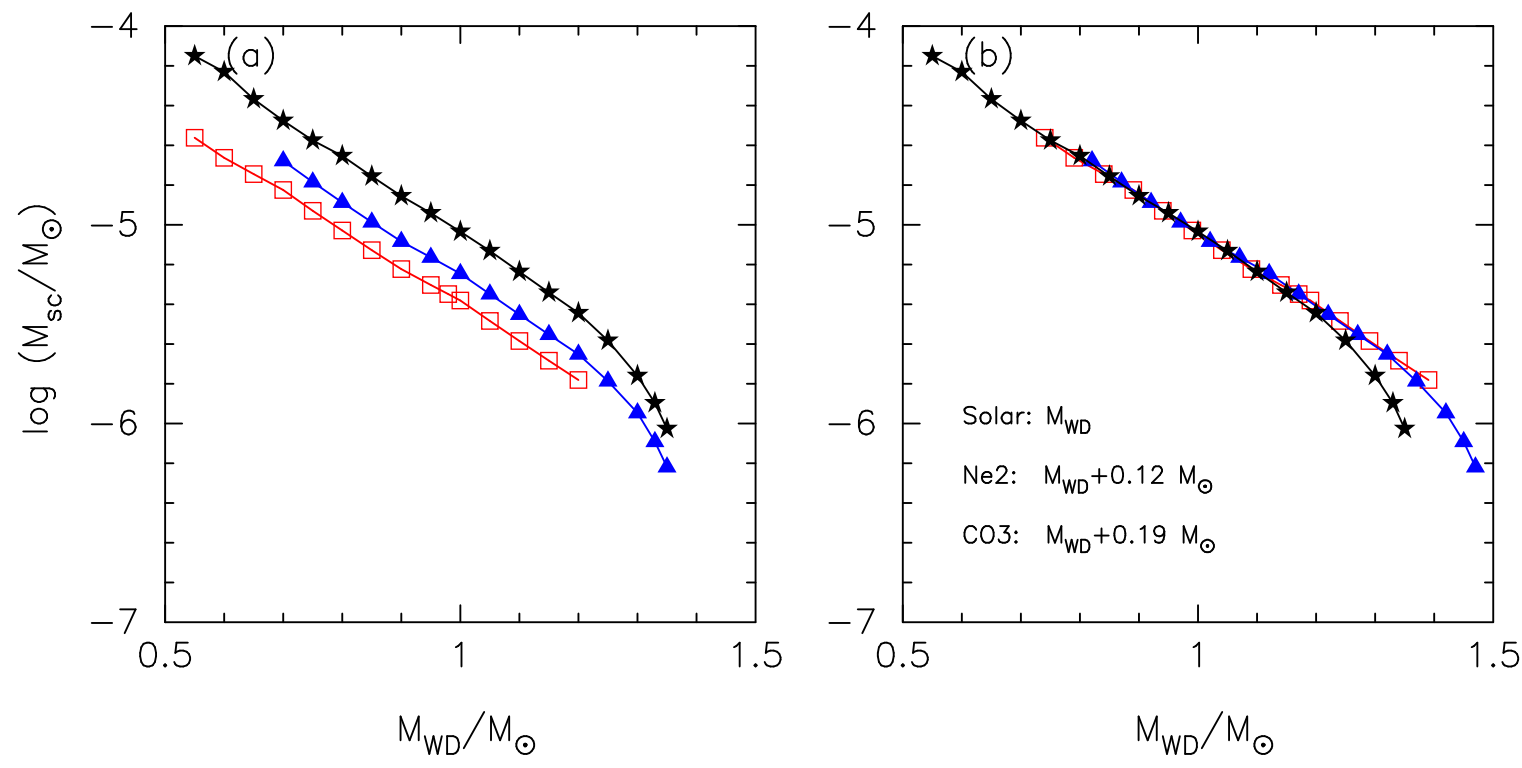

Figure 7. (a) The scaling mass $M_{\mathrm{sc}}$ against the WD mass $M_{\mathrm{WD}}$ for various chemical compositions. The filled black stars connected by a black line show the models in Table 3 (solar composition). The filled blue triangles connected by a blue line denote the models in Table 2 (Ne nova 2). The unfilled red squares connected by a red line correspond to the models in Table 1 (CO nova 3). The CO3 has $X=0.45, Y=0.18, Z=0.02, X_{\mathrm{C}}=0.15$, and $X_{\mathrm{O}}=0.20$ by mass weight. The Ne2 is composed of $X=0.55, Y=0.30, Z=0.02, X_{\mathrm{O}}=0.10$, and $X_{\mathrm{Ne}}=0.03$. (b) Same as in panel (a), but we shift the WD masses of the chemical composition $\mathrm{Ne} 2$ by $+0.12 M_{\odot}$, and of $\mathrm{CO} 3$ by $+0.19 M_{\odot}$ as plotted in the figure. These three lines overlap well until $1.2 M_{\odot}$.

sistent with the equi-recurrence period lines of $t_{\mathrm{rec}}=30$ and $100 \mathrm{yr}$ in Figure 6. We give three reasons for this exceptional case. The first point is that the present theory cannot be applicable to recurrent novae because Equation (32) is not valid for $x_{0} \lesssim 2$ (below the yellow line) as mentioned in Section 3. The second point is that the mass accretion rate is probably larger than $\dot{M}_{\text {acc }} \gtrsim 3 \times 10^{-8} M_{\odot} \mathrm{yr}^{-1}$ in recurrent novae. This means that the WD core is hot and its radius is larger than that of a cold core as mentioned in Section 2.2. We must calculate wind solutions assuming the large WD radius depending on the mass accretion rate. The third point is that a hot helium layer develops underneath a hydrogen-rich envelope in recurrent novae (e.g., Kato et al. 2017b). This possibly affects the thermal state of hydrogen-rich envelope. We must take into account at least these three effects for recurrent novae. The calculation is so complicated that we leave it to near future.

\section{DISCUSSION}

We have obtained the relation between the peak $V$ brightness $M_{V, \max }$ and the rate of decline $t_{3}$ (or $t_{2}$ ) in Section 4.2 for solar composition $(X=0.70, Y=0.28$, and $Z=0.02$ by mass weight). However, it has been reported that nova ejecta are enriched by heavy elements such as carbon, oxygen, and neon (e.g., Gehrz et al.
1998). Here, we discuss the effect of enrichment in heavy elements.

Kato \& Hachisu (1994) calculated nova models for various chemical compositions. We have already tabulated the scaling masses $M_{\mathrm{sc}}$ for three cases, $\mathrm{CO}$ nova 2 (CO2), $\mathrm{CO}$ nova $3(\mathrm{CO} 3)$, and $\mathrm{CO}$ nova $4(\mathrm{CO} 4)$ in Table 1 and two cases, Ne nova $2(\mathrm{Ne} 2)$ and Ne nova 3 (Ne3) in Table 2. The CO3 chemical composition has $X=0.45, Y=0.18, Z=0.02, X_{\mathrm{C}}=0.15$, and $X_{\mathrm{O}}=0.20$ by mass weight. The Ne2 chemical composition is composed of $X=0.55, Y=0.30, Z=0.02$, $X_{\mathrm{O}}=0.10$, and $X_{\mathrm{Ne}}=0.03$. We regard the CO3 to be a typical chemical composition for $\mathrm{CO}$ novae and the $\mathrm{Ne} 2$ to be a typical one for neon novae. The $M_{\mathrm{sc}}$ for the $\mathrm{CO} 3$ and Ne2 are plotted in Figure 7(a) together with the solar composition case in Table 3.

If the scaling mass is the same for two different chemical compositions, their timescales are also the same as inferred from Equation (11). We shift the lines of CO3 and $\mathrm{Ne} 2$ in Figure $7(\mathrm{a})$ toward the right by $+0.19 M_{\odot}$ and $+0.12 M_{\odot}$, respectively, in Figure 7(b). Then, the three lines (CO3, Ne2, and Solar) almost overlap with each other at least until $M_{\mathrm{WD}}=1.2 M_{\odot}$. This means that, for the CO3 case, the timescale $\left(t_{3}\right)$ and the peak $V$ brightness $\left(M_{V, \max }\right)$ of $M_{\mathrm{WD}, \mathrm{CO} 3}+0.19 M_{\odot}$ are the

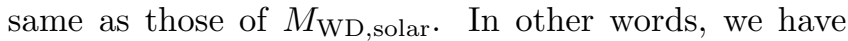
the same MMRD relation between the $\mathrm{CO} 3$ and solar 


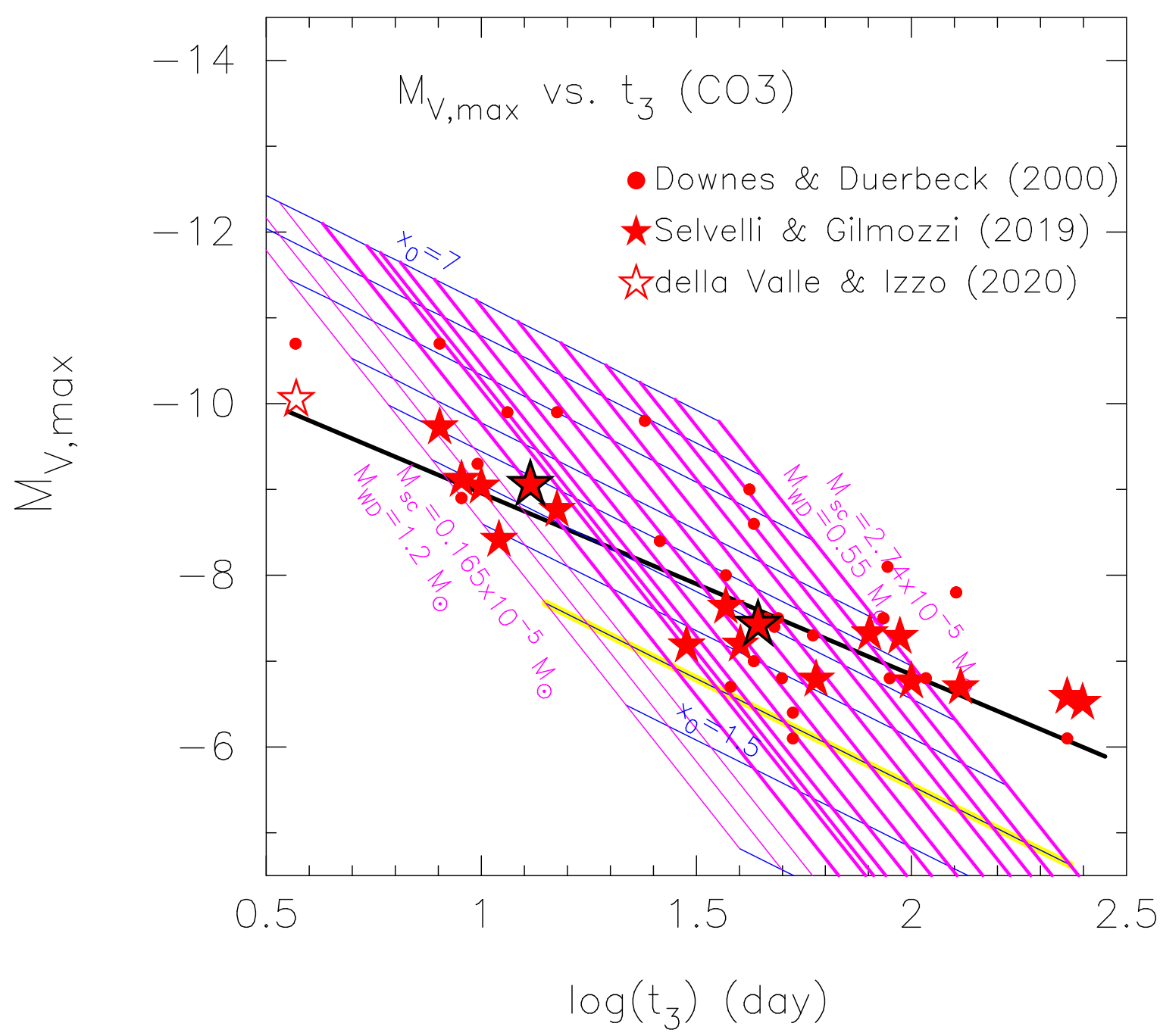

Figure 8. Same as Figure 5, but for the chemical composition of CO nova 3 (CO3). Each scaling mass (magenta line) corresponds to the WD mass, from right to left, $0.55 M_{\odot}$ to $1.2 M_{\odot}$ by $0.05 M_{\odot}$ step except $0.98 M_{\odot}$, the same as those tabulated in Table $1(\mathrm{CO} 3)$. The thick solid magenta lines of equi- $M_{\mathrm{sc}}$ (or equi- $M_{\mathrm{WD}}$ ) correspond to the WD mass region for $\mathrm{CO}$ novae $\left(M_{\mathrm{WD}} \leq 1.05 M_{\odot}\right)$.

compositions at

$$
M_{\mathrm{WD}, \mathrm{CO} 3}=M_{\mathrm{WD}, \mathrm{solar}}-0.19 M_{\odot},
$$

and between the Ne2 and solar at

$$
M_{\mathrm{WD}, \mathrm{Ne} 2}=M_{\mathrm{WD}, \mathrm{solar}}-0.12 M_{\odot} .
$$

This difference in the WD mass for different chemical compositions appears in the WD mass estimate from the light curve fitting (e.g., Hachisu \& Kato 2016). To confirm these WD mass relations, we plot the $\left(\log t_{3}\right)-$ $M_{V \text {, max }}$ diagrams for the CO3 and Ne2 cases in Figures 8 and 9, respectively, which are the same as Figure 5 but for different set of $M_{\mathrm{sc}}$.

To better understand these WD mass relations, we examine the case of V533 Her (Nova Her 1963). Selvelli \& Gilmozzi (2019) obtained $t_{3}=44 \pm 2$ days and $M_{V, \max }=-7.42 \pm 0.22$. The MMRD point of V533 Her is the lower-right outlined red star in Figures 5 and 6 . The position is on the $M_{\mathrm{WD} \text {, solar }}=$ $0.99 \pm 0.03 M_{\odot}$ and $t_{\text {rec }}=4000 \pm 1000 \mathrm{yr}$ in Figure 6. If we assume the chemical composition to be CO3 for V533 Her, we obtained the WD mass of $M_{\mathrm{WD}, \mathrm{CO} 3}=$ $M_{\mathrm{WD} \text {,solar }}-0.19 M_{\odot}=0.80 \pm 0.03 M_{\odot}$. For the Ne2 case, we obtain $M_{\mathrm{WD}, \mathrm{Ne} 2}=M_{\mathrm{WD} \text {,solar }}-0.12 M_{\odot}=$ $0.87 \pm 0.03 M_{\odot}$. We can confirm that these two WD masses are reasonable in Figures 8 and 9, respectively.

For the case of $M_{\mathrm{WD} \text {,solar }} \gtrsim 1.2 M_{\odot}$, the WD mass difference between $M_{\mathrm{WD} \text {,solar }}$ and $M_{\mathrm{WD}, \mathrm{CO} 3}$ or between $M_{\mathrm{WD}, \text { solar }}$ and $M_{\mathrm{WD}, \mathrm{Ne} 2}$ for the same $M_{\mathrm{sc}}$ becomes smaller as shown in Figure 7(a). Then, the WD mass difference should be measured directly from Figure 7(a). We examine the case of GK Per (Nova Per 1901). Selvelli \& Gilmozzi (2019) obtained $t_{3}=13 \pm 1$ days 


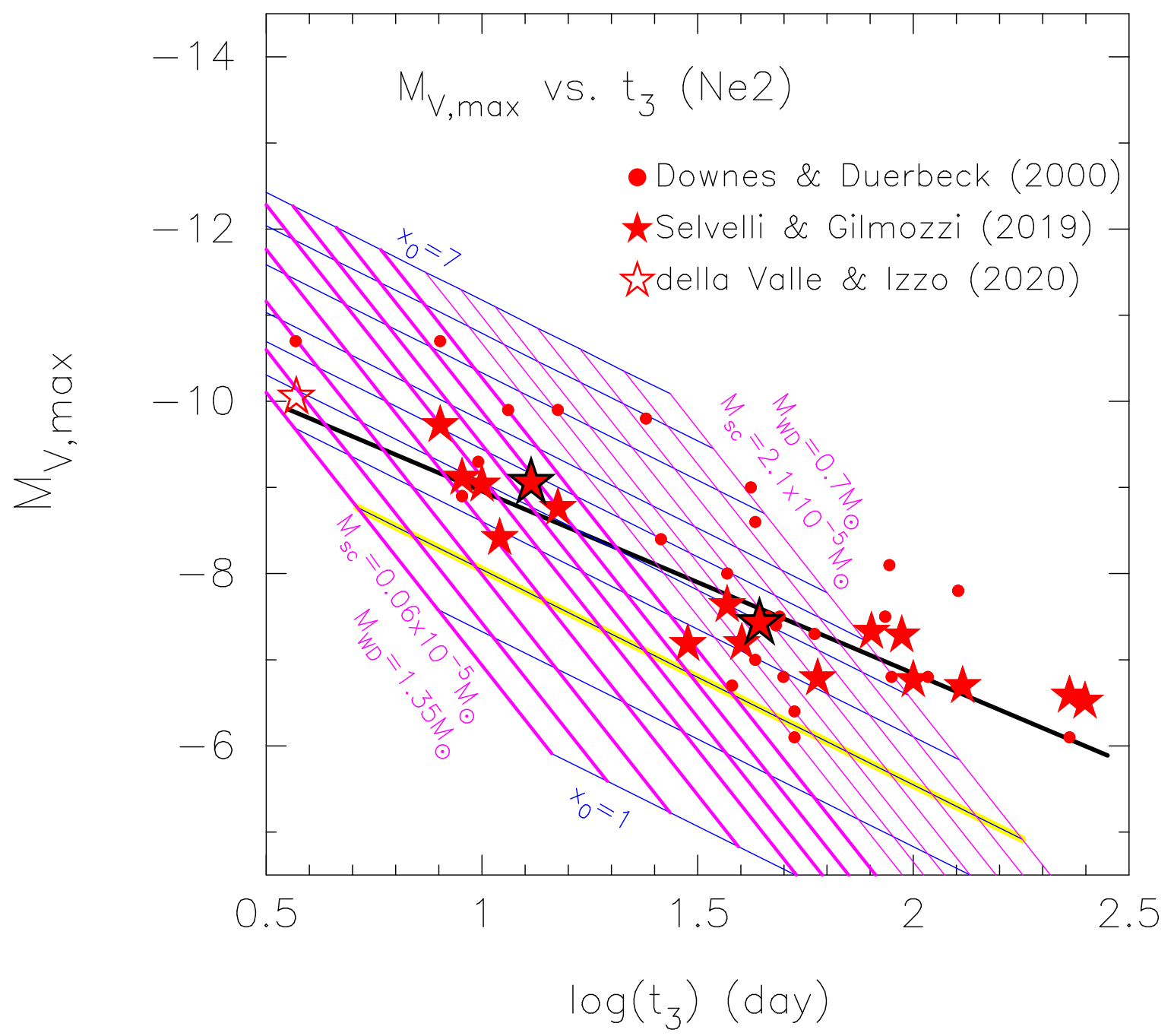

Figure 9. Same as Figure 5, but for the chemical composition of Ne nova 2 (Ne2). Each scaling mass (magenta line) corresponds to the WD mass, from right to left, $0.7 M_{\odot}$ to $1.35 M_{\odot}$ by $0.05 M_{\odot}$ step except $1.33 M_{\odot}$, the same as those tabulated in Table $2(\mathrm{Ne} 2)$. The thick solid magenta lines of equi- $M_{\mathrm{sc}}$ (or equi- $M_{\mathrm{WD}}$ ) correspond to the WD mass region for neon novae $\left(M_{\mathrm{WD}} \geq 1.05 M_{\odot}\right)$.

and $M_{V, \max }=-9.05 \pm 0.16$. The MMRD point is located at/near $M_{\mathrm{WD} \text {, solar }}=1.21 M_{\odot}$ and $t_{\mathrm{rec}}=8 \times 10^{4} \mathrm{yr}$ in Figure 6. If we assume the CO3 chemical composition for GK Per, the WD mass difference is estimated to be $0.17 M_{\odot}$ at $M_{\mathrm{WD} \text {, solar }}=1.21 M_{\odot}$ from Figure $7(\mathrm{a})$. Then, we have $M_{\mathrm{WD}, \mathrm{CO} 3}=M_{\mathrm{WD} \text {, solar }}-0.17 M_{\odot}=$ $1.04 M_{\odot}$. For the Ne2 case, we obtain the difference of $0.10 M_{\odot}$ and the WD mass of $M_{\mathrm{WD}, \mathrm{Ne} 2}=$ $M_{\mathrm{WD} \text {,solar }}-0.10 M_{\odot}=1.11 M_{\odot}$. We can also confirm that these two WD masses are reasonable in Figures 8 and 9 , respectively.

Hachisu \& Kato (2007) estimated the WD mass to be $M_{\mathrm{WD}, \mathrm{Ne} 2}=1.15 \pm 0.05 M_{\odot}$ for GK Per based on the nova model light curve fitting. This is approximately consistent with the above estimate from the MMRD point. On the other hand, Hachisu \& Kato (2019a) estimated the WD mass of V533 Her to be $M_{\mathrm{WD}, \mathrm{Ne} 2}=$
$1.03 \pm 0.05 M_{\odot}$ from the nova model light curve fitting. This value is larger than the above estimate from the MMRD relation, $M_{\mathrm{WD}, \mathrm{Ne} 2}=0.87 \pm 0.03 M_{\odot}$. This is partly because observational $V$ data are very poor near the peak and there are jitters with a $0.5 \mathrm{mag}$ amplitude at 2-mag decay from the peak (see, e.g., Figure 33 of Hachisu \& Kato 2019a).

\section{CONCLUSIONS}

Hachisu \& Kato (2006, 2010) constructed free-free emission model light curves of novae based on the optically-thick wind theory (Kato \& Hachisu 1994). These light curves provided good fit to those of many observed novae. Hachisu \& Kato (2006) also showed that the theoretical nova light curves are homologous against a normalized time $\tau=t / f_{\mathrm{s}}$ and can be expressed with one-parameter family of the timescaling factor $f_{\mathrm{s}}$. 
We find the new parameter set of $\left(M_{\mathrm{sc}}, x\right)$ equivalent to $\left(f_{\mathrm{s}}, \tau\right)$. Using this equivalent conversion, we propose a theory for the maximum magnitude versus rate of decline (MMRD) relation for novae based on the universal decline law. It should be noted that our MMRD relations from the universal decline law are applicable only to specific light curves of novae such as S-types defined by Strope et al. (2010). Our main conclusions are as follows:

1. We adopt the dimensionless envelope mass $x \equiv$ $M_{\mathrm{env}} / M_{\mathrm{sc}}$, as the parameter that describes the nova light curves. The peak $V$ brightness is expressed by an analytic form of $x_{0}$ and $M_{\mathrm{sc}}$, i.e., Equation (33), where $x_{0}$ is the ratio of the initial envelope mass and $M_{\mathrm{sc}}$, $x_{0}=M_{\mathrm{env}, 0} / M_{\mathrm{sc}}$. The decline rate of $t_{3}$ (or $t_{2}$ ) is also calculated from an analytic formula of $x_{0}$ and $M_{\mathrm{sc}}$, i.e., Equation (32) (or Equation (31)).

2. The scaling masses $M_{\mathrm{sc}}$ are defined by the envelope mass having a wind mass-loss rate of $\log \left(\dot{M}_{\text {wind }} / M_{\odot} \mathrm{yr}^{-1}\right)=-4.7$, which are summarized in Tables 1, 2, and 3 for various WD masses and chemical compositions (taken from Kato \& Hachisu 1994), and $x_{0}, M_{V, \max }, t_{2}$, and $t_{3}$ are tabulated in Table 4 for various WD masses and mass accretion rates of solar abundance material (taken from Kato et al. 2014, with additional calculation for the present work).

3. We plot our model MMRD relations for the same WD mass, same mass accretion rate, and same recurrence time (solar composition). The theoretical range of MMRD for expected nova parameters well constrains the MMRD points of observed novae obtained by Downes \& Duerbeck (2000) and Selvelli \& Gilmozzi (2019) except the very long $t_{3} \gtrsim 200$ days region. The WD mass ranges mainly from $1.35 M_{\odot}$ to $0.5 M_{\odot}$. The mass accretion rate is typically between $3 \times 10^{-8} M_{\odot} \mathrm{yr}^{-1}$ and $1 \times 10^{-11} M_{\odot} \mathrm{yr}^{-1}$ centered at $5 \times 10^{-9} M_{\odot} \mathrm{yr}^{-1}$ in the longer $t_{3}$ region of $t_{3}>30$ days. The recurrence time is typically between $300 \mathrm{yr}$ and $10^{6} \mathrm{yr}$.

4. From the MMRD point of an observed nova, we are able to broadly specify the WD mass and mass accretion rate. The main trend in the observed MMRD distribution corresponds to the mass accretion rate of $\dot{M}_{\text {acc }} \sim 5 \times 10^{-9} M_{\odot} \mathrm{yr}^{-1}$ with the different WD masses in the longer $t_{3}$ region of $t_{3}>30$ days. The scatter of nova MMRD points from the main trend line can be attributed to a large scatter of the observed mass accretion rates from that of the main trend defined by $\dot{M}_{\text {acc }} \sim 5 \times 10^{-9} M_{\odot} \mathrm{yr}^{-1}$. Thus, the global trend of an MMRD relation does exist, but its scatter is too large for it to be a precision distance indicator of individual novae.

5. In the shorter $t_{3}$ region of $t_{3}<30$ days, the main trend MMRD relation traverses the four lines of mass-accretion rates, $\dot{M}_{\text {acc }}=3 \times 10^{-9} M_{\odot} \mathrm{yr}^{-1}$, $10^{-9} M_{\odot} \mathrm{yr}^{-1}, 10^{-10} M_{\odot} \mathrm{yr}^{-1}$, and $10^{-11} M_{\odot} \mathrm{yr}^{-1}$. In general, the smaller the $t_{3}$ time, the smaller the $\dot{M}_{\text {acc }}$. The recurrence time is typically between $t_{\mathrm{rec}}=10000$ yr and $10^{6} \mathrm{yr}$.

6. If we divide the data of Selvelli \& Gilmozzi (2019) into two groups by the brightness of $M_{V, \max }=-8.0$, the upper brighter group broadly correspond to cataclysmic variables below the period gap $\left(\dot{M}_{\text {acc }} \sim 10^{-10} M_{\odot} \mathrm{yr}^{-1}\right)$ while the lower fainter group correspond to binaries above the period gap $\left(\dot{M}_{\text {acc }} \sim 5 \times 10^{-9} M_{\odot} \mathrm{yr}^{-1}\right)$.

7. The lower the mass accretion rate, the larger the ignition mass for a given WD mass and chemical composition. Thus, the lower the mass accretion rate, the brighter the $V$ peak of a nova. There is a $M_{V} \sim-10.4$ mag cap for the maximum brightness if we limit the mass accretion rate above $\dot{M}_{\text {acc }} \geq 1 \times 10^{-11} M_{\odot} \mathrm{yr}^{-1}$ (e.g., Knigge et al. 2011). This explains that the brightest peaks of novae are at/around $M_{V} \sim-10.4$. Thus, we clarified the reason for the brightness cap $\left(M_{V} \sim-10.4\right)$ of classical novae, which is constrained by the lowest mass-accretion rate of $\dot{M}_{\mathrm{acc}} \sim 1 \times 10^{-11} M_{\odot} \mathrm{yr}^{-1}$.

8. Finally, we discussed the effect of enrichment of heavy elements in nova ejecta. The enrichment has the same effect as the WD mass decrease. For example, the difference between the abundances of solar and $\mathrm{Ne} 2$ novae is $0.12 M_{\odot}$, i.e., $M_{\mathrm{WD}, \text { solar }}=M_{\mathrm{WD}, \mathrm{Ne} 2}+0.12 M_{\odot}$, for the same $M_{V \text {,max }}$ and $t_{3}$.

\section{ACKNOWLEDGMENTS}

I.H. and M.K. thank Department of Astronomy, San Diego State University, for the warm hospitality, during which we initiated the present work. We are grateful to the anonymous referee for useful comments, which improved the manuscript. 


\section{APPENDIX}

\section{A. NOVA IGNITION MODELS}

We have calculated the MMRD points, $M_{V, \max }$ and $t_{3}$ (or $t_{2}$ ), based on the result of Kato et al. (2014) for various WD masses and mass accretion rates (solar abundance) together with additional calculation that expanded the parameter range of mass-accretion rate for the present work. Corresponding parameters are the WD mass, $M_{\mathrm{WD}}$, mass-accretion rate, $\dot{M}_{\text {acc }}$, accumulation mass, $M_{\text {acc }}$ (Section 4.2.1), scaling mass, $M_{\mathrm{sc}}$ (Section 2.2), parameter $x_{0}$ (Sections 2.2 and 2.3), peak absolute $V$ magnitude, $M_{V, \max }$ (Equation (33)), times $t_{2}, t_{3}$ (Section 3, Equation (32)), and the recurrence time, $t_{\text {rec }}$ (Section 4.2, Figure 6).

Table 4. MMRD Relation for Kato et al.'s Model ${ }^{a}$

\begin{tabular}{|c|c|c|c|c|c|c|c|c|}
\hline $\begin{array}{l}M_{\mathrm{WD}} \\
\left(M_{\odot}\right)\end{array}$ & $\begin{array}{c}\dot{M}_{\mathrm{acc}} \\
\left(M_{\odot} \mathrm{yr}^{-1}\right)\end{array}$ & $\begin{array}{l}M_{\mathrm{acc}} \\
\left(M_{\odot}\right)\end{array}$ & $\begin{array}{c}M_{\mathrm{sc}} \\
\left(M_{\odot}\right)\end{array}$ & $x_{0}$ & $M_{V, \max }$ & $\begin{array}{c}t_{2} \\
\text { (days) }\end{array}$ & $\begin{array}{c}t_{3} \\
\text { (days) }\end{array}$ & $\begin{array}{l}t_{\mathrm{rec}} \\
(\mathrm{yr})\end{array}$ \\
\hline 0.6 & $1.0 \mathrm{E}-11$ & $2.52 \mathrm{E}-4$ & $5.88 \mathrm{E}-5$ & 4.56 & -7.20 & 78.6 & 146. & $2.52 \mathrm{E}+7$ \\
\hline 0.6 & $3.0 \mathrm{E}-11$ & $2.42 \mathrm{E}-4$ & $5.88 \mathrm{E}-5$ & 4.39 & -7.04 & 83.2 & 154. & $8.08 \mathrm{E}+6$ \\
\hline 0.6 & $5.0 \mathrm{E}-11$ & $2.39 \mathrm{E}-4$ & $5.88 \mathrm{E}-5$ & 4.33 & -6.99 & 84.9 & 157. & $4.78 \mathrm{E}+6$ \\
\hline 0.6 & $1.0 \mathrm{E}-10$ & $2.37 \mathrm{E}-4$ & $5.88 \mathrm{E}-5$ & 4.31 & -6.97 & 85.6 & 159. & $2.37 \mathrm{E}+6$ \\
\hline 0.6 & $3.0 \mathrm{E}-10$ & $2.31 \mathrm{E}-4$ & $5.88 \mathrm{E}-5$ & 4.21 & -6.87 & 88.8 & 164. & $7.71 \mathrm{E}+5$ \\
\hline 0.6 & $1.0 \mathrm{E}-9$ & $2.17 \mathrm{E}-4$ & $5.88 \mathrm{E}-5$ & 3.97 & -6.62 & 97.0 & 180. & $2.17 \mathrm{E}+5$ \\
\hline 0.6 & $1.6 \mathrm{E}-9$ & $2.07 \mathrm{E}-4$ & $5.88 \mathrm{E}-5$ & 3.79 & -6.44 & 104. & 192. & $1.29 \mathrm{E}+5$ \\
\hline 0.6 & $3.0 \mathrm{E}-9$ & $1.91 \mathrm{E}-4$ & $5.88 \mathrm{E}-5$ & 3.52 & -6.12 & 116. & 215. & $6.35 \mathrm{E}+4$ \\
\hline 0.6 & $5.0 \mathrm{E}-9$ & $1.75 \mathrm{E}-4$ & $5.88 \mathrm{E}-5$ & 3.24 & -5.79 & 131. & 243. & $3.49 \mathrm{E}+4$ \\
\hline 0.6 & $1.0 \mathrm{E}-8$ & $1.48 \mathrm{E}-4$ & $5.88 \mathrm{E}-5$ & 2.78 & -5.16 & 165. & 306. & $1.48 \mathrm{E}+4$ \\
\hline 0.6 & $1.6 \mathrm{E}-8$ & $1.32 \mathrm{E}-4$ & $5.88 \mathrm{E}-5$ & 2.52 & -4.74 & 192. & 356. & 8235 \\
\hline 0.6 & $2.0 \mathrm{E}-8$ & $1.25 \mathrm{E}-4$ & $5.88 \mathrm{E}-5$ & 2.41 & -4.56 & 205. & 380. & 6269. \\
\hline 0.6 & $3.0 \mathrm{E}-8$ & $1.15 \mathrm{E}-4$ & $5.88 \mathrm{E}-5$ & 2.24 & -4.26 & 229. & 424. & 3846. \\
\hline 0.7 & $1.0 \mathrm{E}-11$ & $1.72 \mathrm{E}-4$ & $3.35 \mathrm{E}-5$ & 5.44 & -8.54 & 34.4 & 63.6 & $1.72 \mathrm{E}+7$ \\
\hline 0.7 & $3.0 \mathrm{E}-11$ & $1.63 \mathrm{E}-4$ & $3.35 \mathrm{E}-5$ & 5.17 & -8.32 & 37.1 & 68.8 & $5.44 \mathrm{E}+6$ \\
\hline 0.7 & $5.0 \mathrm{E}-11$ & $1.60 \mathrm{E}-4$ & $3.35 \mathrm{E}-5$ & 5.06 & -8.24 & 38.3 & 70.9 & $3.19 \mathrm{E}+6$ \\
\hline 0.7 & $1.0 \mathrm{E}-10$ & $1.55 \mathrm{E}-4$ & $3.35 \mathrm{E}-5$ & 4.93 & -8.13 & 39.8 & 73.7 & $1.55 \mathrm{E}+6$ \\
\hline 0.7 & $3.0 \mathrm{E}-10$ & $1.48 \mathrm{E}-4$ & $3.35 \mathrm{E}-5$ & 4.70 & -7.93 & 42.8 & 79.2 & $4.92 \mathrm{E}+5$ \\
\hline 0.7 & $1.0 \mathrm{E}-9$ & $1.35 \mathrm{E}-4$ & $3.35 \mathrm{E}-5$ & 4.33 & -7.59 & 48.4 & 89.6 & $1.35 \mathrm{E}+5$ \\
\hline 0.7 & $3.0 \mathrm{E}-9$ & $1.10 \mathrm{E}-4$ & $3.35 \mathrm{E}-5$ & 3.59 & -6.82 & 64.1 & 119. & $3.68 \mathrm{E}+4$ \\
\hline 0.7 & $1.0 \mathrm{E}-8$ & $8.81 \mathrm{E}-5$ & $3.35 \mathrm{E}-5$ & 2.92 & -5.98 & 87.2 & 161. & 8808. \\
\hline 0.7 & $3.0 \mathrm{E}-8$ & $6.47 \mathrm{E}-5$ & $3.35 \mathrm{E}-5$ & 2.22 & -4.85 & 131. & 243. & 2156. \\
\hline 0.7 & $5.0 \mathrm{E}-8$ & $5.68 \mathrm{E}-5$ & $3.35 \mathrm{E}-5$ & 1.99 & -4.39 & 155. & 288. & 1136. \\
\hline 0.7 & $6.0 \mathrm{E}-8$ & $5.47 \mathrm{E}-5$ & $3.35 \mathrm{E}-5$ & 1.93 & -4.25 & 163. & 302. & 911. \\
\hline 0.8 & $1.0 \mathrm{E}-11$ & $1.18 \mathrm{E}-4$ & $2.22 \mathrm{E}-5$ & 5.56 & -9.07 & 22.1 & 40.9 & $1.18 \mathrm{E}+7$ \\
\hline 0.8 & $3.0 \mathrm{E}-11$ & $1.10 \mathrm{E}-4$ & $2.22 \mathrm{E}-5$ & 5.20 & -8.80 & 24.4 & 45.2 & $3.68 \mathrm{E}+6$ \\
\hline 0.8 & $5.0 \mathrm{E}-11$ & $1.07 \mathrm{E}-4$ & $2.22 \mathrm{E}-5$ & 5.06 & -8.68 & 25.4 & 47.1 & $2.15 \mathrm{E}+6$ \\
\hline 0.8 & $1.0 \mathrm{E}-10$ & $1.06 \mathrm{E}-4$ & $2.22 \mathrm{E}-5$ & 4.98 & -8.62 & 26.1 & 48.3 & $1.06 \mathrm{E}+6$ \\
\hline 0.8 & $3.0 \mathrm{E}-10$ & $1.00 \mathrm{E}-4$ & $2.22 \mathrm{E}-5$ & 4.74 & -8.41 & 28.1 & 52.0 & $3.34 \mathrm{E}+5$ \\
\hline 0.8 & $1.0 \mathrm{E}-9$ & $9.18 \mathrm{E}-5$ & $2.22 \mathrm{E}-5$ & 4.37 & -8.07 & 31.8 & 58.8 & $9.18 \mathrm{E}+4$ \\
\hline 0.8 & $1.6 \mathrm{E}-9$ & $8.70 \mathrm{E}-5$ & $2.22 \mathrm{E}-5$ & 4.15 & -7.86 & 34.3 & 63.5 & $5.44 \mathrm{E}+4$ \\
\hline 0.8 & $3.0 \mathrm{E}-9$ & $7.90 \mathrm{E}-5$ & $2.22 \mathrm{E}-5$ & 3.79 & -7.49 & 39.3 & 72.8 & $2.63 \mathrm{E}+4$ \\
\hline 0.8 & $5.0 \mathrm{E}-9$ & $7.21 \mathrm{E}-5$ & $2.22 \mathrm{E}-5$ & 3.48 & -7.14 & 44.7 & 82.7 & $1.44 \mathrm{E}+4$ \\
\hline 0.8 & $1.0 \mathrm{E}-8$ & $6.01 \mathrm{E}-5$ & $2.22 \mathrm{E}-5$ & 2.94 & -6.44 & 57.6 & 107. & 6006. \\
\hline 0.8 & $1.6 \mathrm{E}-8$ & $5.19 \mathrm{E}-5$ & $2.22 \mathrm{E}-5$ & 2.57 & -5.89 & 70.3 & 130. & 3244 . \\
\hline 0.8 & $3.0 \mathrm{E}-8$ & $4.29 \mathrm{E}-5$ & $2.22 \mathrm{E}-5$ & 2.17 & -5.18 & 90.9 & 168. & 1430. \\
\hline
\end{tabular}

Table 4 continued 
HACHISU ET AL.

Table 4 (continued)

\begin{tabular}{|c|c|c|c|c|c|c|c|c|}
\hline $\begin{array}{l}M_{\mathrm{WD}} \\
\left(M_{\odot}\right)\end{array}$ & $\begin{array}{c}M_{\mathrm{acc}} \\
\left(M_{\odot} \mathrm{yr}^{-1}\right)\end{array}$ & $\begin{array}{l}M_{\text {acc }} \\
\left(M_{\odot}\right)\end{array}$ & $\begin{array}{c}M_{\mathrm{Sc}} \\
\left(M_{\odot}\right)\end{array}$ & $x_{0}$ & $M_{V, \max }$ & $\begin{array}{c}t_{2} \\
\text { (days) }\end{array}$ & $\begin{array}{c}t_{3} \\
\text { (days) }\end{array}$ & $\begin{array}{l}t_{\text {rec }} \\
(\mathrm{yr})\end{array}$ \\
\hline 0.8 & $5.0 \mathrm{E}-8$ & $3.67 \mathrm{E}-5$ & $2.22 \mathrm{E}-5$ & 1.89 & -4.61 & 112 . & 207. & 734 . \\
\hline 0.8 & $7.0 \mathrm{E}-8$ & $3.34 \mathrm{E}-5$ & $2.22 \mathrm{E}-5$ & 1.74 & -4.27 & 127. & 234 & 477. \\
\hline 0.8 & $7.5 \mathrm{E}-8$ & $3.28 \mathrm{E}-5$ & $2.22 \mathrm{E}-5$ & 1.71 & -4.21 & 130. & 240 . & 437. \\
\hline 0.9 & $1.0 \mathrm{E}-11$ & $7.93 \mathrm{E}-5$ & $1.39 \mathrm{E}-5$ & 5.90 & -9.83 & 12.7 & 23.4 & $7.93 \mathrm{E}+6$ \\
\hline 0.9 & $3.0 \mathrm{E}-11$ & $7.29 \mathrm{E}-5$ & $1.39 \mathrm{E}-5$ & 5.44 & -9.49 & 14.3 & 26.5 & $2.43 \mathrm{E}+6$ \\
\hline 0.9 & $5.0 \mathrm{E}-11$ & $7.04 \mathrm{E}-5$ & $1.39 \mathrm{E}-5$ & 5.26 & -9.35 & 15.0 & 27.8 & $1.41 \mathrm{E}+6$ \\
\hline 0.9 & $1.0 \mathrm{E}-10$ & $6.74 \mathrm{E}-5$ & $1.39 \mathrm{E}-5$ & 5.05 & -9.18 & 16.0 & 29.6 & $6.74 \mathrm{E}+5$ \\
\hline 0.9 & $3.0 \mathrm{E}-10$ & $6.31 \mathrm{E}-5$ & $1.39 \mathrm{E}-5$ & 4.74 & -8.92 & 17.6 & 32.6 & $2.10 \mathrm{E}+5$ \\
\hline 0.9 & $1.0 \mathrm{E}-9$ & $5.66 \mathrm{E}-5$ & $1.39 \mathrm{E}-5$ & 4.27 & -8.49 & 20.5 & 38.0 & $5.66 \mathrm{E}+4$ \\
\hline 0.9 & $3.0 \mathrm{E}-9$ & $4.84 \mathrm{E}-5$ & $1.39 \mathrm{E}-5$ & 3.69 & -7.88 & 25.6 & 47.5 & $1.61 \mathrm{E}+4$ \\
\hline 0.9 & $1.0 \mathrm{E}-8$ & $3.87 \mathrm{E}-5$ & $1.39 \mathrm{E}-5$ & 2.99 & -7.01 & 35.2 & 65.1 & 3867. \\
\hline 0.9 & $3.0 \mathrm{E}-8$ & $2.72 \mathrm{E}-5$ & $1.39 \mathrm{E}-5$ & 2.17 & -5.69 & 56.9 & 105. & 908. \\
\hline 0.9 & $5.0 \mathrm{E}-8$ & $2.30 \mathrm{E}-5$ & $1.39 \mathrm{E}-5$ & 1.86 & -5.06 & 71.6 & 133. & 459. \\
\hline 0.9 & $7.0 \mathrm{E}-8$ & $2.06 \mathrm{E}-5$ & $1.39 \mathrm{E}-5$ & 1.69 & -4.66 & 82.7 & 153. & 294 . \\
\hline 0.9 & $9.0 \mathrm{E}-8$ & $1.90 \mathrm{E}-5$ & $1.39 \mathrm{E}-5$ & 1.58 & -4.38 & 91.6 & 170 . & 212 . \\
\hline 0.9 & $1.0 \mathrm{E}-7$ & $1.84 \mathrm{E}-5$ & $1.39 \mathrm{E}-5$ & 1.53 & -4.27 & 95.5 & 177. & 184. \\
\hline 0.9 & $1.1 \mathrm{E}-7$ & $1.79 \mathrm{E}-5$ & $1.39 \mathrm{E}-5$ & 1.50 & -4.17 & 99.0 & 183. & 163. \\
\hline 1.0 & $1.0 \mathrm{E}-11$ & $5.07 \mathrm{E}-5$ & $9.25 \mathrm{E}-6$ & 5.68 & -10.11 & 8.89 & 16.5 & $5.07 \mathrm{E}+6$ \\
\hline 1.0 & $3.0 \mathrm{E}-11$ & $4.59 \mathrm{E}-5$ & $9.25 \mathrm{E}-6$ & 5.16 & -9.71 & 10.3 & 19.1 & $1.53 \mathrm{E}+6$ \\
\hline 1.0 & $5.0 \mathrm{E}-11$ & $4.41 \mathrm{E}-5$ & $9.25 \mathrm{E}-6$ & 4.96 & -9.55 & 10.9 & 20.2 & $8.81 \mathrm{E}+5$ \\
\hline 1.0 & $1.0 \mathrm{E}-10$ & $4.30 \mathrm{E}-5$ & $9.25 \mathrm{E}-6$ & 4.85 & -9.46 & 11.3 & 20.9 & $4.30 \mathrm{E}+5$ \\
\hline 1.0 & $3.0 \mathrm{E}-10$ & $4.00 \mathrm{E}-5$ & $9.25 \mathrm{E}-6$ & 4.53 & -9.18 & 12.5 & 23.2 & $1.33 \mathrm{E}+5$ \\
\hline 1.0 & $1.0 \mathrm{E}-9$ & $3.63 \mathrm{E}-5$ & $9.25 \mathrm{E}-6$ & 4.12 & -8.79 & 14.4 & 26.6 & $3.63 \mathrm{E}+4$ \\
\hline 1.0 & $1.6 \mathrm{E}-9$ & $3.44 \mathrm{E}-5$ & $9.25 \mathrm{E}-6$ & 3.92 & -8.58 & 15.5 & 28.7 & $2.15 \mathrm{E}+4$ \\
\hline 1.0 & $3.0 \mathrm{E}-9$ & $3.12 \mathrm{E}-5$ & $9.25 \mathrm{E}-6$ & 3.57 & -8.20 & 17.9 & 33.1 & $1.04 \mathrm{E}+4$ \\
\hline 1.0 & $5.0 \mathrm{E}-9$ & $2.85 \mathrm{E}-5$ & $9.25 \mathrm{E}-6$ & 3.27 & -7.84 & 20.3 & 37.7 & 5689. \\
\hline 1.0 & $1.0 \mathrm{E}-8$ & $2.39 \mathrm{E}-5$ & $9.25 \mathrm{E}-6$ & 2.78 & -7.16 & 26.0 & 48.2 & 2386. \\
\hline 1.0 & $1.6 \mathrm{E}-8$ & $2.05 \mathrm{E}-5$ & $9.25 \mathrm{E}-6$ & 2.41 & -6.58 & 32.2 & 59.6 & 1280. \\
\hline 1.0 & $3.0 \mathrm{E}-8$ & $1.67 \mathrm{E}-5$ & $9.25 \mathrm{E}-6$ & 2.00 & -5.80 & 42.6 & 78.9 & 555. \\
\hline 1.0 & $5.0 \mathrm{E}-8$ & $1.39 \mathrm{E}-5$ & $9.25 \mathrm{E}-6$ & 1.69 & -5.12 & 54.6 & 101. & 277 . \\
\hline 1.0 & $1.0 \mathrm{E}-7$ & $1.09 \mathrm{E}-5$ & $9.25 \mathrm{E}-6$ & 1.37 & -4.25 & 75.0 & 139. & 109. \\
\hline 1.0 & $1.2 \mathrm{E}-7$ & $1.02 \mathrm{E}-5$ & $9.25 \mathrm{E}-6$ & 1.30 & -4.03 & 81.1 & 150. & 85.1 \\
\hline 1.1 & $1.0 \mathrm{E}-11$ & $3.01 \mathrm{E}-5$ & $5.81 \mathrm{E}-6$ & 5.36 & -10.37 & 6.10 & 11.3 & $3.01 \mathrm{E}+6$ \\
\hline 1.1 & $3.0 \mathrm{E}-11$ & $2.68 \mathrm{E}-5$ & $5.81 \mathrm{E}-6$ & 4.78 & -9.91 & 7.23 & 13.4 & $8.93 \mathrm{E}+5$ \\
\hline 1.1 & $5.0 \mathrm{E}-11$ & $2.56 \mathrm{E}-5$ & $5.81 \mathrm{E}-6$ & 4.58 & -9.73 & 7.72 & 14.3 & $5.12 \mathrm{E}+5$ \\
\hline 1.1 & $1.0 \mathrm{E}-10$ & $2.49 \mathrm{E}-5$ & $5.81 \mathrm{E}-6$ & 4.46 & -9.62 & 8.03 & 14.9 & $2.49 \mathrm{E}+5$ \\
\hline 1.1 & $3.0 \mathrm{E}-10$ & $2.31 \mathrm{E}-5$ & $5.81 \mathrm{E}-6$ & 4.14 & -9.31 & 8.98 & 16.6 & $7.69 \mathrm{E}+4$ \\
\hline 1.1 & $1.0 \mathrm{E}-9$ & $2.09 \mathrm{E}-5$ & $5.81 \mathrm{E}-6$ & 3.76 & -8.92 & 10.4 & 19.2 & $2.09 \mathrm{E}+4$ \\
\hline 1.1 & $1.6 \mathrm{E}-9$ & $1.98 \mathrm{E}-5$ & $5.81 \mathrm{E}-6$ & 3.57 & -8.70 & 11.2 & 20.8 & $1.23 \mathrm{E}+4$ \\
\hline 1.1 & $3.0 \mathrm{E}-9$ & $1.79 \mathrm{E}-5$ & $5.81 \mathrm{E}-6$ & 3.26 & -8.32 & 12.9 & 23.8 & 5976. \\
\hline 1.1 & $5.0 \mathrm{E}-9$ & $1.64 \mathrm{E}-5$ & $5.81 \mathrm{E}-6$ & 2.99 & -7.97 & 14.6 & 27.1 & 3277. \\
\hline 1.1 & $1.0 \mathrm{E}-8$ & $1.38 \mathrm{E}-5$ & $5.81 \mathrm{E}-6$ & 2.55 & -7.31 & 18.6 & 34.5 & 1381. \\
\hline 1.1 & $1.6 \mathrm{E}-8$ & $1.19 \mathrm{E}-5$ & $5.81 \mathrm{E}-6$ & 2.22 & -6.73 & 23.0 & 42.5 & 742 \\
\hline 1.1 & $3.0 \mathrm{E}-8$ & $9.62 \mathrm{E}-6$ & $5.81 \mathrm{E}-6$ & 1.83 & -5.94 & 30.6 & 56.7 & 321. \\
\hline 1.1 & $5.0 \mathrm{E}-8$ & $7.95 \mathrm{E}-6$ & $5.81 \mathrm{E}-6$ & 1.54 & -5.23 & 39.6 & 73.4 & 159. \\
\hline 1.1 & $1.0 \mathrm{E}-7$ & $6.13 \mathrm{E}-6$ & $5.81 \mathrm{E}-6$ & 1.23 & -4.29 & 55.8 & 103. & 61.3 \\
\hline 1.1 & $1.6 \mathrm{E}-7$ & $5.14 \mathrm{E}-6$ & $5.81 \mathrm{E}-6$ & 1.06 & -3.68 & 69.7 & 129. & 32.2 \\
\hline 1.2 & $1.0 \mathrm{E}-11$ & $1.54 \mathrm{E}-5$ & $3.60 \mathrm{E}-6$ & 4.42 & -10.10 & 5.04 & 9.34 & $1.54 \mathrm{E}+6$ \\
\hline 1.2 & $3.0 \mathrm{E}-11$ & $1.35 \mathrm{E}-5$ & $3.60 \mathrm{E}-6$ & 3.90 & -9.59 & 6.08 & 11.3 & $4.50 \mathrm{E}+5$ \\
\hline
\end{tabular}

Table 4 continued 
Table 4 (continued)

\begin{tabular}{|c|c|c|c|c|c|c|c|c|}
\hline $\begin{array}{l}M_{\mathrm{WD}} \\
\left(M_{\odot}\right)\end{array}$ & $\begin{array}{c}M_{\mathrm{acc}} \\
\left(M_{\odot} \mathrm{yr}^{-1}\right)\end{array}$ & $\begin{array}{l}M_{\text {acc }} \\
\left(M_{\odot}\right)\end{array}$ & $\begin{array}{c}M_{\mathrm{Sc}} \\
\left(M_{\odot}\right)\end{array}$ & $x_{0}$ & $M_{V, \max }$ & $\begin{array}{c}t_{2} \\
\text { (days) }\end{array}$ & $\begin{array}{c}t_{3} \\
\text { (days) }\end{array}$ & $\begin{array}{l}t_{\text {rec }} \\
(\mathrm{yr})\end{array}$ \\
\hline 1.2 & $5.0 \mathrm{E}-11$ & $1.28 \mathrm{E}-5$ & $3.60 \mathrm{E}-6$ & 3.70 & -9.37 & 6.59 & 12.2 & $2.55 \mathrm{E}+5$ \\
\hline 1.2 & $1.0 \mathrm{E}-10$ & $1.24 \mathrm{E}-5$ & $3.60 \mathrm{E}-6$ & 3.61 & -9.26 & 6.85 & 12.7 & $1.24 \mathrm{E}+5$ \\
\hline 1.2 & $3.0 \mathrm{E}-10$ & $1.14 \mathrm{E}-5$ & $3.60 \mathrm{E}-6$ & 3.33 & -8.93 & 7.73 & 14.3 & $3.81 \mathrm{E}+4$ \\
\hline 1.2 & $1.0 \mathrm{E}-9$ & $1.03 \mathrm{E}-5$ & $3.60 \mathrm{E}-6$ & 3.02 & -8.53 & 8.94 & 16.6 & $1.03 \mathrm{E}+4$ \\
\hline 1.2 & $1.6 \mathrm{E}-9$ & $9.77 \mathrm{E}-6$ & $3.60 \mathrm{E}-6$ & 2.87 & -8.31 & 9.67 & 17.9 & 6107. \\
\hline 1.2 & $3.0 \mathrm{E}-9$ & $8.89 \mathrm{E}-6$ & $3.60 \mathrm{E}-6$ & 2.62 & -7.94 & 11.1 & 20.5 & 2962. \\
\hline 1.2 & $5.0 \mathrm{E}-9$ & $8.12 \mathrm{E}-6$ & $3.60 \mathrm{E}-6$ & 2.41 & -7.59 & 12.6 & 23.4 & 1624. \\
\hline 1.2 & $1.0 \mathrm{E}-8$ & $6.85 \mathrm{E}-6$ & $3.60 \mathrm{E}-6$ & 2.05 & -6.94 & 15.9 & 29.5 & 685 \\
\hline 1.2 & $1.6 \mathrm{E}-8$ & $5.89 \mathrm{E}-6$ & $3.60 \mathrm{E}-6$ & 1.79 & -6.37 & 19.6 & 36.3 & 368. \\
\hline 1.2 & $3.0 \mathrm{E}-8$ & $4.76 \mathrm{E}-6$ & $3.60 \mathrm{E}-6$ & 1.47 & -5.57 & 26.2 & 48.6 & 159. \\
\hline 1.2 & $5.0 \mathrm{E}-8$ & $3.90 \mathrm{E}-6$ & $3.60 \mathrm{E}-6$ & 1.24 & -4.85 & 34.1 & 63.2 & 78.0 \\
\hline 1.2 & $1.0 \mathrm{E}-7$ & $2.97 \mathrm{E}-6$ & $3.60 \mathrm{E}-6$ & 0.978 & -3.88 & 48.5 & 89.9 & 29.7 \\
\hline 1.2 & $1.6 \mathrm{E}-7$ & $2.45 \mathrm{E}-6$ & $3.60 \mathrm{E}-6$ & 0.832 & -3.21 & 61.8 & 114. & 15.3 \\
\hline 1.2 & $1.8 \mathrm{E}-7$ & $2.33 \mathrm{E}-6$ & $3.60 \mathrm{E}-6$ & 0.800 & -3.05 & 65.6 & 122. & 12.9 \\
\hline 1.25 & $1.0 \mathrm{E}-11$ & $9.93 \mathrm{E}-6$ & $2.61 \mathrm{E}-6$ & 3.95 & -9.99 & 4.33 & 8.01 & $9.93 \mathrm{E}+5$ \\
\hline 1.25 & $3.0 \mathrm{E}-11$ & $8.61 \mathrm{E}-6$ & $2.61 \mathrm{E}-6$ & 3.44 & -9.42 & 5.32 & 9.84 & $2.87 \mathrm{E}+5$ \\
\hline 1.25 & $5.0 \mathrm{E}-11$ & $8.15 \mathrm{E}-6$ & $2.61 \mathrm{E}-6$ & 3.27 & -9.20 & 5.75 & 10.7 & $1.63 \mathrm{E}+5$ \\
\hline 1.25 & $1.0 \mathrm{E}-10$ & $7.92 \mathrm{E}-6$ & $2.61 \mathrm{E}-6$ & 3.18 & -9.09 & 5.99 & 11.1 & $7.92 \mathrm{E}+4$ \\
\hline 1.25 & $3.0 \mathrm{E}-10$ & $7.27 \mathrm{E}-6$ & $2.61 \mathrm{E}-6$ & 2.93 & -8.75 & 6.78 & 12.6 & $2.42 \mathrm{E}+4$ \\
\hline 1.25 & $1.0 \mathrm{E}-9$ & $6.57 \mathrm{E}-6$ & $2.61 \mathrm{E}-6$ & 2.66 & -8.35 & 7.84 & 14.5 & 6565 \\
\hline 1.25 & $1.6 \mathrm{E}-9$ & $6.21 \mathrm{E}-6$ & $2.61 \mathrm{E}-6$ & 2.52 & -8.13 & 8.48 & 15.7 & 3881. \\
\hline 1.25 & $3.0 \mathrm{E}-9$ & $5.65 \mathrm{E}-6$ & $2.61 \mathrm{E}-6$ & 2.31 & -7.77 & 9.69 & 17.9 & 1884. \\
\hline 1.25 & $5.0 \mathrm{E}-9$ & $5.16 \mathrm{E}-6$ & $2.61 \mathrm{E}-6$ & 2.12 & -7.42 & 11.0 & 20.4 & 1031. \\
\hline 1.25 & $1.0 \mathrm{E}-8$ & $4.34 \mathrm{E}-6$ & $2.61 \mathrm{E}-6$ & 1.81 & -6.76 & 14.0 & 25.9 & 434. \\
\hline 1.25 & $1.6 \mathrm{E}-8$ & $3.75 \mathrm{E}-6$ & $2.61 \mathrm{E}-6$ & 1.58 & -6.20 & 17.1 & 31.7 & 234 . \\
\hline 1.25 & $3.0 \mathrm{E}-8$ & $3.02 \mathrm{E}-6$ & $2.61 \mathrm{E}-6$ & 1.30 & -5.40 & 22.9 & 42.4 & 101. \\
\hline 1.25 & $5.0 \mathrm{E}-8$ & $2.49 \mathrm{E}-6$ & $2.61 \mathrm{E}-6$ & 1.10 & -4.70 & 29.6 & 54.8 & 49.8 \\
\hline 1.25 & $1.0 \mathrm{E}-7$ & $1.87 \mathrm{E}-6$ & $2.61 \mathrm{E}-6$ & 0.858 & -3.69 & 42.8 & 79.2 & 18.7 \\
\hline 1.25 & $1.6 \mathrm{E}-7$ & $1.52 \mathrm{E}-6$ & $2.61 \mathrm{E}-6$ & 0.722 & -2.98 & 55.3 & 103. & 9.47 \\
\hline 1.25 & $2.0 \mathrm{E}-7$ & $1.37 \mathrm{E}-6$ & $2.61 \mathrm{E}-6$ & 0.668 & -2.65 & 62.3 & 115. & 6.86 \\
\hline 1.3 & $1.0 \mathrm{E}-11$ & $5.59 \mathrm{E}-6$ & $1.74 \mathrm{E}-6$ & 3.35 & -9.75 & 3.69 & 6.84 & $5.59 \mathrm{E}+5$ \\
\hline 1.3 & $3.0 \mathrm{E}-11$ & $4.83 \mathrm{E}-6$ & $1.74 \mathrm{E}-6$ & 2.91 & -9.17 & 4.56 & 8.45 & $1.61 \mathrm{E}+5$ \\
\hline 1.3 & $5.0 \mathrm{E}-11$ & $4.55 \mathrm{E}-6$ & $1.74 \mathrm{E}-6$ & 2.75 & -8.94 & 4.96 & 9.18 & $9.11 \mathrm{E}+4$ \\
\hline 1.3 & $1.0 \mathrm{E}-10$ & $4.40 \mathrm{E}-6$ & $1.74 \mathrm{E}-6$ & 2.66 & -8.80 & 5.21 & 9.66 & $4.40 \mathrm{E}+4$ \\
\hline 1.3 & $3.0 \mathrm{E}-10$ & $4.03 \mathrm{E}-6$ & $1.74 \mathrm{E}-6$ & 2.45 & -8.46 & 5.90 & 10.9 & $1.34 \mathrm{E}+4$ \\
\hline 1.3 & $1.0 \mathrm{E}-9$ & $3.65 \mathrm{E}-6$ & $1.74 \mathrm{E}-6$ & 2.23 & -8.07 & 6.80 & 12.6 & 3646. \\
\hline 1.3 & $1.5 \mathrm{E}-9$ & $3.48 \mathrm{E}-6$ & $1.74 \mathrm{E}-6$ & 2.13 & -7.89 & 7.26 & 13.5 & 2319. \\
\hline 1.3 & $3.0 \mathrm{E}-9$ & $3.14 \mathrm{E}-6$ & $1.74 \mathrm{E}-6$ & 1.94 & -7.49 & 8.39 & 15.5 & 1046. \\
\hline 1.3 & $1.0 \mathrm{E}-8$ & $2.41 \mathrm{E}-6$ & $1.74 \mathrm{E}-6$ & 1.52 & -6.49 & 12.1 & 22.3 & 241 \\
\hline 1.3 & $3.0 \mathrm{E}-8$ & $1.69 \mathrm{E}-6$ & $1.74 \mathrm{E}-6$ & 1.10 & -5.16 & 19.6 & 36.2 & 56.2 \\
\hline 1.3 & $1.0 \mathrm{E}-7$ & $1.02 \mathrm{E}-6$ & $1.74 \mathrm{E}-6$ & 0.720 & -3.40 & 37.1 & 68.7 & 10.2 \\
\hline 1.3 & $1.6 \mathrm{E}-7$ & $8.27 \mathrm{E}-7$ & $1.74 \mathrm{E}-6$ & 0.609 & -2.71 & 47.7 & 88.3 & 5.17 \\
\hline 1.3 & $2.0 \mathrm{E}-7$ & $7.44 \mathrm{E}-7$ & $1.74 \mathrm{E}-6$ & 0.561 & -2.38 & 53.9 & 99.8 & 3.72 \\
\hline 1.3 & $2.2 \mathrm{E}-7$ & $7.12 \mathrm{E}-7$ & $1.74 \mathrm{E}-6$ & 0.543 & -2.24 & 56.6 & 105. & 3.24 \\
\hline 1.35 & $1.0 \mathrm{E}-11$ & $2.38 \mathrm{E}-6$ & $9.40 \mathrm{E}-7$ & 2.64 & -9.43 & 2.86 & 5.30 & $2.38 \mathrm{E}+5$ \\
\hline 1.35 & $3.0 \mathrm{E}-11$ & $2.06 \mathrm{E}-6$ & $9.40 \mathrm{E}-7$ & 2.30 & -8.86 & 3.51 & 6.50 & $6.86 \mathrm{E}+4$ \\
\hline 1.35 & $5.0 \mathrm{E}-11$ & $1.95 \mathrm{E}-6$ & $9.40 \mathrm{E}-7$ & 2.19 & -8.66 & 3.79 & 7.01 & $3.91 \mathrm{E}+4$ \\
\hline 1.35 & $1.0 \mathrm{E}-10$ & $1.85 \mathrm{E}-6$ & $9.40 \mathrm{E}-7$ & 2.08 & -8.45 & 4.09 & 7.57 & $1.85 \mathrm{E}+4$ \\
\hline 1.35 & $3.0 \mathrm{E}-10$ & $1.71 \mathrm{E}-6$ & $9.40 \mathrm{E}-7$ & 1.93 & -8.14 & 4.57 & 8.47 & 5700 \\
\hline 1.35 & $1.0 \mathrm{E}-9$ & $1.54 \mathrm{E}-6$ & $9.40 \mathrm{E}-7$ & 1.75 & -7.74 & 5.28 & 9.78 & 1544. \\
\hline
\end{tabular}

Table 4 continued 
Table 4 (continued)

\begin{tabular}{|c|c|c|c|c|c|c|c|c|}
\hline $\begin{array}{l}M_{\mathrm{WD}} \\
\left(M_{\odot}\right)\end{array}$ & $\begin{array}{c}M_{\mathrm{acc}} \\
\left(M_{\odot} \mathrm{yr}^{-1}\right)\end{array}$ & $\begin{array}{l}M_{\mathrm{acc}} \\
\left(M_{\odot}\right)\end{array}$ & $\begin{array}{c}M_{\mathrm{SC}} \\
\left(M_{\odot}\right)\end{array}$ & $\overline{x_{0}}$ & $M_{V, \max }$ & $\begin{array}{c}t_{2} \\
\text { (days) }\end{array}$ & $\begin{array}{c}t_{3} \\
\text { (days) }\end{array}$ & $\begin{array}{l}t_{\text {rec }} \\
(\mathrm{yr})\end{array}$ \\
\hline 1.35 & $1.6 \mathrm{E}-9$ & $1.46 \mathrm{E}-6$ & $9.40 \mathrm{E}-7$ & 1.66 & -7.53 & 5.70 & 10.6 & 914. \\
\hline 1.35 & $3.0 \mathrm{E}-9$ & $1.34 \mathrm{E}-6$ & $9.40 \mathrm{E}-7$ & 1.53 & -7.18 & 6.47 & 12.0 & 445. \\
\hline 1.35 & $5.0 \mathrm{E}-9$ & $1.22 \mathrm{E}-6$ & $9.40 \mathrm{E}-7$ & 1.41 & -6.84 & 7.33 & 13.6 & 244. \\
\hline 1.35 & $1.0 \mathrm{E}-8$ & $1.03 \mathrm{E}-6$ & $9.40 \mathrm{E}-7$ & 1.21 & -6.21 & 9.21 & 17.1 & 103. \\
\hline 1.35 & $1.6 \mathrm{E}-8$ & $9.06 \mathrm{E}-7$ & $9.40 \mathrm{E}-7$ & 1.07 & -5.72 & 11.0 & 20.4 & 56.6 \\
\hline 1.35 & $3.0 \mathrm{E}-8$ & $7.33 \mathrm{E}-7$ & $9.40 \mathrm{E}-7$ & 0.889 & -4.94 & 14.6 & 27.1 & 24.4 \\
\hline 1.35 & $5.0 \mathrm{E}-8$ & $6.03 \mathrm{E}-7$ & $9.40 \mathrm{E}-7$ & 0.751 & -4.24 & 18.8 & 34.9 & 12.1 \\
\hline 1.35 & $1.0 \mathrm{E}-7$ & $4.50 \mathrm{E}-7$ & $9.40 \mathrm{E}-7$ & 0.587 & -3.23 & 27.2 & 50.4 & 4.50 \\
\hline 1.35 & $1.6 \mathrm{E}-7$ & $3.62 \mathrm{E}-7$ & $9.40 \mathrm{E}-7$ & 0.494 & -2.52 & 35.3 & 65.3 & 2.26 \\
\hline 1.35 & $2.0 \mathrm{E}-7$ & $3.25 \mathrm{E}-7$ & $9.40 \mathrm{E}-7$ & 0.455 & -2.18 & 39.9 & 73.9 & 1.62 \\
\hline 1.35 & $2.5 \mathrm{E}-7$ & $2.91 \mathrm{E}-7$ & $9.40 \mathrm{E}-7$ & 0.419 & -1.84 & 45.1 & 83.6 & 1.17 \\
\hline
\end{tabular}

\section{REFERENCES}

Cao, Y., Kasliwal, M. M., Neill, J. D., et al. 2012, ApJ, 752, 133

Capaccioli, M., della Valle, M., D'Onofrio, M., Rosino, L. 1990, ApJ, 360, 63

Chen, H.-L., Woods, T. E., Yungelson, L. R., et al. 2019, MNRAS, 490, 1678

Cohen, J. G. 1985, ApJ, 292, 90

della Valle, M., \& Izzo, L. 2020, The Astronomy and

Astrophysics Review, 28, 3

della Valle, M., \& Livio, M. 1995, ApJ, 452, 704

di Paolantonio, A., Patriarca, R., \& Tempesti, P. 1981, Inf.

Bull. Variable Stars, 1913, 1

Downes, R. A., \& Duerbeck, H. W. 2000, AJ, 120, 2007

Ennis, D., Becklin, E. E., Beckwith, S., et al. 1977, ApJ, 214,478

Gallagher, J. S., \& Ney, E. P. 1976, ApJ, 204, L35

Gehrz, R. D., Truran, J. W., Williams, R. E., \& Starrfield, S. 1998, PASP, 110, 3

Hachisu, I., \& Kato, M. 2006, ApJS, 167, 59

Hachisu, I., \& Kato, M. 2007, ApJ, 662, 552

Hachisu, I., \& Kato, M. 2010, ApJ, 709, 680

Hachisu, I., \& Kato, M. 2015, ApJ, 798, 76

Hachisu, I., \& Kato, M. 2016, ApJ, 816, 26

Hachisu, I., \& Kato, M. 2017, in Proceedings of the

Palermo Workshop 2017 on "The Golden Age of

Cataclysmic Variables and Related Objects - IV", ed. F.

Giovannelli et al. (Trieste: SISSA PoS), 315, 47

Hachisu, I., \& Kato, M. 2018, ApJS, 237, 4

Hachisu, I., \& Kato, M. 2019a, ApJS, 241, 4

Hachisu, I., \& Kato, M. 2019b, ApJS, 242, 18

Hachisu, I., Saio, H., \& Kato, M. 2016, ApJ, 824, 22
Kasliwal, M. M., Cenko, S. B., Kulkarni, S. R., et al. 2011, ApJ, 735, 94

Kato, M., \& Hachisu, I., 1994, ApJ, 437, 802

Kato, M., Hachisu, I., \& Saio, H. 2017a, in Proceedings of the Palermo Workshop 2017 on "The Golden Age of Cataclysmic Variables and Related Objects - IV", ed. F. Giovannelli et al. (Trieste: SISSA PoS), 315, 56 (arXiv: 1711.01529)

Kato, M., Saio, H., Hachisu, I., \& Nomoto, K. 2014, ApJ, 793, 136

Kato, M., Saio, H., \& Hachisu, I. 2017b, ApJ, 844, 143

Knigge, C., Baraffe, I., \& Patterson, J. 2011, ApJS, 194, 28

Livio, M. 1992, ApJ, 393, 516

Mallama, A. D., \& Skillman, D. R. 1979, PASP, 91, 99

McLaughlin, D. B. 1945, PASP, 57, 69

Nomoto, K. 1982, ApJ, 253, 798

Özdörmez, A., Ege, E., Güver, T., \& Ak, T. 2018, MNRAS, 476, 4162

Prialnik, D., \& Kovetz, A. 1995, ApJ, 445, 789

Schaefer, B. E. 2010, ApJS, 187, 275

Schaefer, B. E. 2018, MNRAS, 481, 3033

Schaefer, B. E., \& Collazzi, A. C. 2010, ApJ, 139, 1831

Schmidt, Th. 1957, Z. Astrophys., 41, 181

Selvelli, P., \& Gilmozzi, R. 2019, A\&A, 622, A186

Shafter, A. W. 2013, AJ, 145, 117

Shafter, A. W., Darnley, M. J., Hornoch, K., et al. 2011, ApJ, 734, 12

Shara, M. M., Doyle, T., Lauer, T. R., et al. 2017, ApJ, 839, 109

Strope, R., Schaefer, B. E., \& Henden, A. A. 2010, AJ, 140, 34 
Townsley, D. M., \& Bildsten, L. 2004, 600, 390
Wolf, W. M., Bildsten, L., Brooks, J., \& Paxton, B. 2013a, ApJ, 777, 136

Wolf, W. M., Bildsten, L., Brooks, J., \& Paxton, B. 2013b, ApJ, 782, 117 (Erratum) 